\title{
Best Selection of Generative Adversarial Networks Hyper-Parameters using Genetic Algorithm
}

Fajr Alarsan ( $\square$ fajr.ib.alarsan@gmail.com )

Higher Institute for Applied Science and Technology https://orcid.org/0000-0001-6057-8339

Mamoon Younes

Damascus university

Research

Keywords: Generative Adversarial Networks (GAN), MNIST dataset, Image 1 synthesis, Generator, Discriminator, Genetic Algorithm

Posted Date: November 6th, 2020

DOl: https://doi.org/10.21203/rs.3.rs-95571/v1

License: (1) This work is licensed under a Creative Commons Attribution 4.0 International License.

Read Full License

Version of Record: A version of this preprint was published at SN Computer Science on May 20th, 2021.

See the published version at https://doi.org/10.1007/s42979-021-00689-3. 


\title{
RESEARCH
}

\section{Best Selection of Generative Adversarial Networks Hyper-Parameters using Genetic Algorithm}

\author{
Fajr Ibrahem Alarsan* (D) and Mamoon Younes
}

Fajr Ibrahem Alarsan: fajr.ib.alarsan@gmail.com, Informatics and Decision Supporting Systems, Higher Institute for Applied Sciences and Technology

Mamoon Younes: Myyounes60@gmail.com, Faculty of Computer and Automation Engineering, Damascuse University

\author{
*Correspondence: \\ fajr.ib.alarsan@gmail.com \\ Informatics and Decision \\ Supporting Systems, Higher \\ Institute for Applied Sciences and \\ Technology, Damascus, Syria \\ Full list of author information is \\ available at the end of the article
}

\begin{abstract}
Generative Adversarial Networks (GANs) are most popular generative frameworks that have achieved compelling performance. They follow an adversarial approach where two deep models generator and discriminator compete with each other In this paper, we propose a Generative Adversarial Network with best hyper-parameters selection to generate fake images for digits number 1 to 9 with generator and train discriminator to decide whereas the generated images are fake or true. Using Genetic Algorithm technique to adapt GAN hyper-parameters, the final method is named GANGA:Generative Adversarial Network with Genetic Algorithm. Anaconda environment with tensorflow library facilitates was used, python as programming language also used with needed libraries. The implementation was done using MNIST dataset to validate our work. The proposed method is to let Genetic algorithm to choose best values of hyper-parameters depending on minimizing a cost function such as a loss function or maximizing accuracy function. GA was used to select values of Learning rate, Batch normalization, Number of neurons and a parameter of Dropout layer.
\end{abstract}

Keywords: Generative Adversarial Networks (GAN), MNIST dataset, Image synthesis, Generator,Discriminator, Genetic Algorithm

\section{Introduction}

Many machine learning systems look at some kind of complicated input (say, an image) and produce a simple output (a categorical label like, "cat" or numeric label like 1, 2, or any other number that represent a class). By contrast, the goal of a generative model is something like the opposite: take a small piece of inputperhaps a few random numbers or vector of noise-and produce a complex output, like an image of a realistic-looking face. A generative adversarial network (GAN) is an especially effective type of generative model, introduced only a few years ago, which has been a subject of intense interest in the machine learning community [1].

The idea of a GAN is creating realistic images from scratch can seem like magic, but GANs use special method to turn a vague, seemingly impossible goal into reality. The method is to use randomness as an ingredient. At a basic level, it would not be very exciting if we build a system that produce the same face each time it ran. Thinking in terms of probabilities, it also helps us translate the problem of generating realistic images into a natural mathematical framework. The system 
should learn about which images are likely to be faces, and which are not. Mathematically, this involves modeling a probability distribution on images, that is, a function that tells us which images are likely to be faces and which are not. This type of problem modeling a function on a high dimensional space is exactly the sort of thing neural networks are made for. GAN must set up this modeling problem as a kind of contest. This is where the "adversarial" part of the name comes from. The key idea is to build not one, but two competing networks: a generator and a discriminator. The generator tries to create random synthetic outputs, while the discriminator tries to tell these apart from real outputs. The hope is that as the two networks will both get better and better with the end result being a generator network that produces realistic outputs. Figure 1 below explains GAN simply:

[Figure 1 about here.]

Generative adversarial networks are neural networks that learn to get samples from a special distribution (the "generative" part of the name) as input, and they do this by setting up a competition (hence "adversarial"). So the main concept behind this project is the generative adversarial network. GAN is about creating stuff and this is hard to compare other deep leaning fields. In other words, Generative adversarial networks (GANs) are deep neural net architectures included of two nets, pitting one against the other

In machine learning, a hyper-parameter is a parameter whose value is used to control the learning process. By contrast, the values of other parameters (typically node weights) are derived via training. Hyper-parameters can be classified as model hyper-parameters, that cannot be inferred while fitting the machine to the training set because they refer to the model selection task, or algorithm hyper-parameters, that in principle have no influence on the performance of the model but affect the speed and quality of the learning process. The choice of hyper-parameters can significantly affect the resulting models performance, but determining good values can be complex [2].

Figure 2 below explains hyper-parameters and default model parameters

[Figure 2 about here.]

46 Hyper-parameter Optimization or Hyper-parameter Tuning can be defined as 47 choosing the right set of values in building a machine learning model, in our case 48 the machine learning model is GAN.

49 Any GAN hyper-parameters can be summarized to:

${ }_{50} \quad$ Learning rate

${ }_{51}$ - Batch size

52 - Number of epochs

53 - Generator optimizer

${ }_{54}$ - Discriminator optimizer

${ }_{55}$ - Number of layers

${ }_{56}$ - Number of units in a dense layer

57 - Activation function

${ }_{58}$ - Loss function 
- Properties such as: keep probability of dropout layer and Batch Normalization momentum

GANs potential is very huge because they can learn to mimic any data. So use of GAN we create worlds similar to our own in any domain: image, anime, news anchor, speech.

\subsection{GAN Applications}

GAN has interesting applications that are commonly used in the industry right now.

- GANs for Image Editing: Most image editing software these days do not give us much flexibility to make creative changes in pictures. For example, let us say we want to change the appearance of a 90-year-old person by changing his/her hairstyle. This can not be done by the current image editing tools out there. Another similar application is image de-raining (or literally removing rainy texture from images [3].

- Using GANs for Security: A constant concern of industrial applications is that they should be robust to cyber attacks. There is a lot of confidential information on the line! GANs are proving to be of immense help here, directly addressing the concern of adversarial attacks. These adversarial attacks use a variety of techniques to fool deep learning architectures. GANs are used to make existing deep learning models more robust to these techniques. How? By creating more such fake examples and training the model to identify them. Pretty clever stuff [4].

- Generating Data with GANs: The availability of data in certain domains is a necessity, especially in domains where training data is needed to model supervision deep learning algorithms. The health care industry comes to mind here. GANs shine again as they can be used to generate synthetic data for supervision. That is right! You know where to go next time you need more data [5].

- GANs for 3D Object Generation: Game designers work countless hours recreating 3D avatars and backgrounds to give them a realistic feel. It certainly takes a lot of effort to create 3D models by imagination. GAN has incredible power to be used to automate the entire process and create 3D models. [6].

There are also other applications, So Gan is very important, interesting, and useful tool to be understood and studied well.

\section{Background and Related work}

There are many works related to GAN hyper-parameters tunning.

In [7], the authors tried to find the appropriate structure more conveniently and efficiently. A method with multi-objective algorithm was proposed to obtain the optimal structure for the GANs. In the proposed method, the non dominated sorting genetic algorithm II (NSGA II) is utilized to optimize the hyper-parameters and structure of deep convolution generative adversarial network (DCGAN). The experiments are conducted on MNIST and Malware datasets demonstrate the efficiency and high performance of proposed method.

In [8], authors proposes the use of Conditional Generative Adversarial Networks (cGANs) for trading strategies calibration and aggregation. They provide a full 
methodology on: (i) the training and selection of a cGAN for time series data; (ii) how each sample is used for strategies calibration; and (iii) how all generated samples can be used for ensemble modeling. They have designed an experiment with multiple trading strategies, encompassing 579 assets. They compared cGAN with an ensemble scheme and model validation methods, both suited for time series. The results suggest that cGANs are a suitable alternative for strategies calibration and combination, providing out performance when the traditional techniques fail to generate any alpha. Their problem can be decomposed into two tasks model validation and hyper-parameter optimization. For each hyper-parameter, they have a space of values, they hope the desire that this space contains the best value of hyper-parameter. Best value will give a max value of accuracy or min value of loss function or error.

In [9], conditional version of Generative Adversarial Networks (cGAN) is used to approximate the true data distribution and generate data for the minority class of various imbalanced datasets. The performance of cGAN is compared against multiple standard oversampling algorithms. They present empirical results that show a significant improvement in the quality of the generated data when cGAN is used as an oversampling algorithm. The hyper-parameters of cGAN are the dimension of the noise space, the hyper-parameters related to the G (Generator) and D (Discriminative) networks architecture as well as their training options. The hyper-parameter tuning of the classifiers and the various oversampling algorithms was done in order to maximize the AUC: Area Under the Curve of the validation set.

In [10], authors propose and study an architectural modification (self-modulation), which improves GAN performance across different data sets, architectures, losses, regularizers, and hyper-parameter settings. They found that self-modulation allows the intermediate feature maps of a generator to change as a function of the input noise vector. While reminiscent of other conditioning techniques, it requires no labeled data. They also observe a relative decrease of 5\% to $35 \%$ in FID (Frechet Inception Distanc). They made a modification to the generator and that leads to improved performance (86\%) of the studied settings.

authors found that most models can reach similar scores with enough hyperparameters optimization and random restarts. They suggested that improvements can arise from a higher computational budget and tuning more than fundamental algorithmic changes. To overcome some limitations of some metrics, they also proposed several data sets on which precision and recall can be computed. Their experimental results suggested that future GAN research should be based on more systematic and objective evaluation procedures.

\section{Our Work}

In this paper, we studied the effects of hyper-parameters in GAN, how the affect the generator and discriminator. So our work is distinguished by:

- Choosing best Learning rate for GAN

- Choosing best dropout keep probability

- Choosing best batch size

- Choosing best number of neurons in dense layers 


\section{Methods}

Here, the concept of our work is explained in details, Genetic algorithm was used to get best values of some hyper-parameters.

\subsection{Genetic Algorithm}

According to [11]. Genetic algorithm (GA) is a metaheuristic ${ }^{\text {a }}$ inspired by the process of natural selection that belongs to the larger class of evolutionary algorithms (EA). Genetic algorithms are commonly used to generate high-quality solutions to optimization and search problems by relying on biologically inspired operators such as mutation, crossover and selection. Genetic Algorithms are used to provide quality solutions for optimization problems and search problems. To get more details about GA, see [12].

\subsection{GAN}

GAN consists of two main part Generator and Discriminator. The training phase of the discriminator and generator are kept separate. In other words, the weights of the generator remain fixed while it produces examples for the discriminator to train on, and vice versa when it is time to train the generator

The discriminator training process is comparable to that of any other neural network. The discriminator classifies both real samples and fake data from the generator. The discriminator loss function penalizes the discriminator for misclassifying a real instance as fake or a fake instance as real, and updates the discriminator's weights via back-propagation, discriminator has loss and accuracy function to be validated.

Similarly, the generator generates samples which are then classified by the discriminator as being fake or real. The results are then fed into a loss function which penalizes the generator for failing to fool the discriminator and back-propagation is used to modify the generator's weights, generator has loss function.

As the generator improves with training, the discriminator performance gets worse because the discriminator fails to distinguish between real and fake. If the generator succeeds perfectly, then the discriminator has a $50 \%$ accuracy (no better than random chance). The later poses a real problem for convergence of the GAN as a whole. If the GAN continues training past the point when the discriminator is giving completely random feedback, then the generator starts to train on junk feedback, and its own performance may be affected. The generator is typically a de-convolutional neural network, and the discriminator is a convolutional neural network [13] when generator is very accurate; i.e its loss function ends to zero, discriminator has bad accuracy and vice versa.

Figure 3 shows Generator (de-convolutional neural network) and Discriminator (convolutional neural network)

[Figure 3 about here.]

\section{3 hyper-parameters tuning}

Tuning process with GA needs a term to be minimization during GA running. From the above description of GAN, we have Generator loss: g_loss, Discriminator loss: 
d_loss and Discriminator accuracy: d_acc

g_loss Minimization $\rightarrow$ d_acc Minimization $\leftrightarrow$ d_loss Maximization

d_acc Maximization $\leftrightarrow$ d_loss Minimization $\rightarrow 5$ g_loss Maximization

\section{Implementation and Results}

\subsection{Programming environment}

According to many references, Anaconda is a free and open-source distribution of the Python and $\mathrm{R}$ programming languages for scientific computing (data science, machine learning applications, data processing, predictive analytics, etc.), that aims to simplify package management and deployment. The distribution includes data science packages suitable for Windows, Linux, and macOS. It is developed and maintained by Anaconda, Inc., which was founded by Peter Wang and Travis Oliphant in 2012. Details about python version is show next

[Figure 4 about here.]

With tensorflow version 1.14.0 and python version 3.7.4. Keras version 2.2.5 was used for implementation. Keras is an open-source library that provides a Python interface for artificial neural networks. Keras acts as an interface for the TensorFlow library. Keras is an API designed for human beings, not machines. Keras follows best practices for reducing cognitive load: it offers consistent and simple APIs, it minimizes the number of user actions required for common use cases, and it provides clear and actionable error messages. It also has extensive documentation and developer guides [13].

\subsection{Basic Model}

Basic Generator in GAN model is show in Figure 5:

[Figure 5 about here.]

while each layer in the generator is shown in the Figure 6:

[Figure 6 about here.]

\section{Basic Discriminator in GAN model is show in Figure 7:}

[Figure 7 about here.]

The Optimizer was Adam: Adaptive Moment Estimation; Adam is an algorithm for first-order gradient-based optimization of stochastic objective functions, based on adaptive estimates of lower-order moments. It works better (faster and more reliably reaching a global minimum) when minimizing the cost function in training [14].

Discriminator and Generator loss function is "binary crossentropy"; Also called Sigmoid Cross-Entropy loss. It is a Sigmoid activation plus a Cross-Entropy loss, it is independent for each vector component (class), meaning that the loss computed for every Discriminator output vector component is not affected by other component values. That is why it is used for multi-label classification; MNIST dataset is multilabel classification, were the insight of an element belonging to a certain class should 
not influence the decision for another class. It is called Binary Cross Entropy Loss because it sets up a binary classification problem between $\mathrm{C}=2$ classes for every class in $\mathrm{C}$.

\subsubsection{Layers Details}

For LeakReLU activation function, Alpha was chosen to be 0.2 , where LeakReLU activation function is as follow:

$$
\begin{aligned}
& f(x)=\alpha * x, \text { if }: x<0 \\
& f(x)=x, \quad \text { if }: \quad x>=0
\end{aligned}
$$

Default value of $\alpha$ is 0.3

For Batch Normalization, momentum is the importance given to the moving average or it is the lag in learning mean and variance, so that noise due to mini-batch can be ignored as described in the equation:

$$
\begin{aligned}
& \mu_{\text {new }}=\beta * \mu_{\text {old }}+(1-\beta) * \mu_{\text {current }} \\
& \sigma_{\text {new }}^{2}=\beta * \sigma_{\text {old }}^{2}+(1-\beta) * \sigma_{\text {current }}^{2} \\
& \beta=\text { momentum }
\end{aligned}
$$

By default, momentum would be set a high value about 0.99 , meaning high lag and slow learning. When batch sizes are small, the no. of steps run will be more. So high momentum will result in slow but steady learning (more lag) of the moving mean. So, in this case, it is helpful. But when the batch size is bigger, as I have used, i.e $5 \mathrm{~K}$ images (out of $50 \mathrm{~K}$ ) in single step, the number of steps is less. Also, the statistics of mini-batch are mostly same as that of the population. At these times, momentum has to be less, so that the mean and variance are updated quickly. Hence a ground rule is that:

- Small batch size $=i$ High Momentum (0.9 to 0.99)

- Big batch size $=i$ Low Momentum (0.6 to 0.85 )

Dropout layer can be also added to Discriminator, dropout refers to dropping out units (both hidden and visible) in a neural network. Dropout refers to ignoring neurons during the training phase of certain set of neurons by a term named Keep Probability, which is chosen at random. By ignoring; i.e. these units are not considered during a particular forward or backward pass [15]

\subsubsection{Genetic algorithm implementation}

Using geneticalgorithm library to implement Genetic algorithm, Table 1 show the main code of implementation

[Table 1 about here.]

dimension refers to number of variables that the GA will find best values of them

variable_type refers to types of variables that the GA will find best values of them 
variable_boundaries refers to min and max of area limits of variables that the GA will find best values of them

Other parameters can be set using algorithm_param [16].

\section{Experiments Results}

\subsection{Tuning for Discriminator Loss}

In this test, mission for GA was to minimize Discriminator Loss, so the returned value from the $\mathrm{f}(\mathrm{X})$ function in GA was the Discriminator Loss (Table 1); We can also minimize the value (1-Discriminator accuracy).

\subsubsection{Learning Rate}

First of all, learning rate was chosen to be tunned, after setting all needed parameters, results gave some values of learning rate that helped to get Discriminator Loss 0 and Discriminator accuracy 100\%. Figure 8 show some results during training:

[Figure 8 about here.]

\section{Filter results for only accuracy $100 \%$ are shown in Figure 9}

[Figure 9 about here.]

max Generator loss was 12.89 and min value was 8.06 .

For max loss of generator, learning rate had some values: 0.013,0.019,0.09,0.08,0.00019, most repeated value was: 0.00019 . For min loss of generator, learning rate had value: 0.3 .

\subsubsection{Other parameters results}

Repeating previous test to get best values of other parameters (keep probability, Dense neurons, batch size), best values are show in next table

[Table 2 about here.]

\subsection{Tuning for Generator Loss \\ In this test, mission for GA was to minimize Generator Loss, so the returned value from the $\mathrm{f}(\mathrm{X})$ function in GA was the Generator Loss (Table 1). \\ 6.2.1 Learning Rate \\ First of all, learning rate was chosen to be tunned, after setting all needed parame- ters, results gave some values of learning rate that helped to get Generator Loss 0. Figure 10 show some results during training:}

[Figure 10 about here.]

Filter results for only loss 0.0 are shown in Figure 11

[Figure 11 about here.]

max Discriminator loss was 7.97 and min value was 4.78 .

For max loss of Discriminator, most repeated value was: 0.171924 . For min loss of generator, learning rate had value: 0.0154 . 


\subsubsection{Other parameters results}

Repeating previous test to get best values of other parameters (keep probability, Dense neurons, batch size), best values are show in next table

[Table 3 about here.]

\section{Figure 12 below shows results during Generator learning when all best parameters} were set

[Figure 12 about here.]

\subsection{Discussion}

From the results, it can be said that GAN with GA (GANGA) is more useful to be used in all fields. In our case, it is applied to MNIST dataset, but it can be implemented to any other dataset such as fingerprints dataset or any other datasets. Once the user has all details about application, GANGA can be applied tp get best parameters. Comparing with [17], the author validate the value 0.0001 for learning rate and 16 for batch size, with seam loss type, max Discriminator accuracy was 96.25\% using ACGAN: Auxiliary classifier generative adversarial network. In our case the Discriminator accuracy was $100 \%$ for learning rate 0.00019984 and batch size 64 and keep probability 0.812 .

\section{Conclusion}

A Generative Adversarial Network is presented in this study to to generate fake images for digits from 1 to 9 , and train it to classify the results into fake or real. Genetic Algorithm was used to select best values for some hyper-parameters of GAN, results showed the importance of GA in selecting hyper-parameters. As a future work, the structure and design of GAN can be edited with best values of hyper-parameters to make GAN as robust as possible.

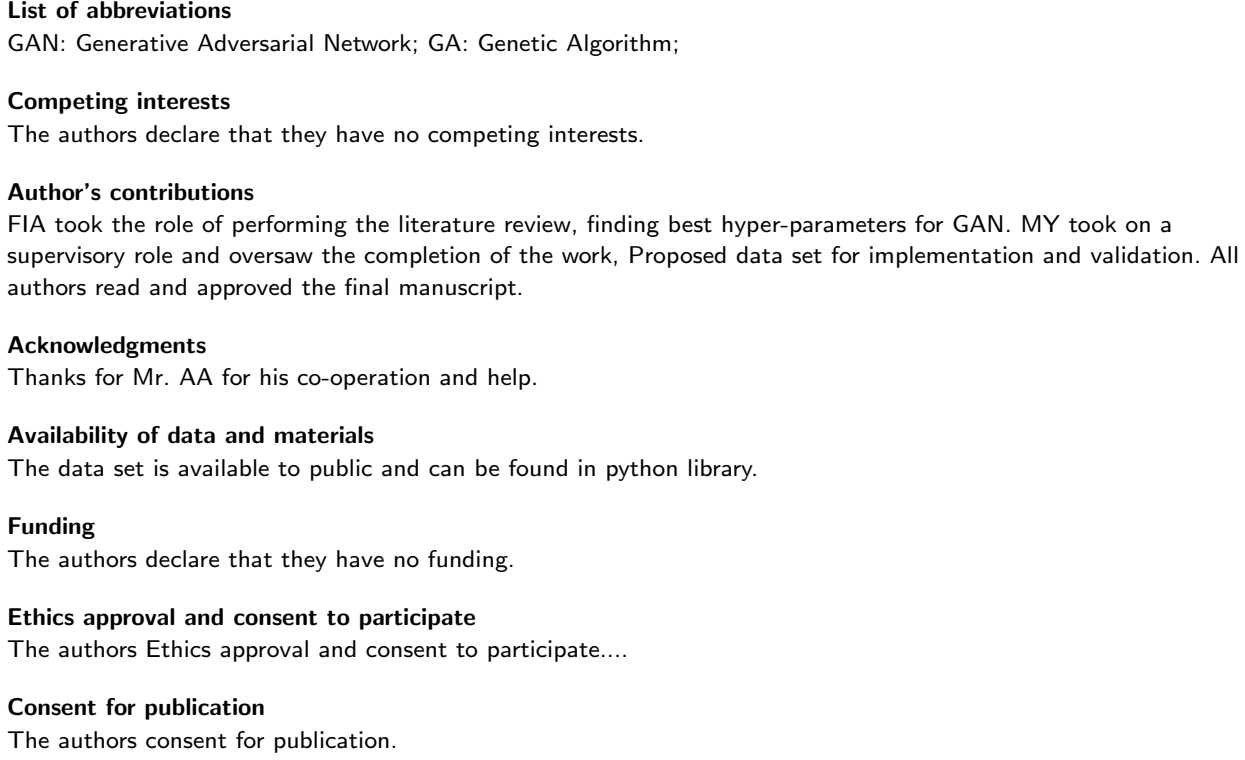




\section{Authors' information}

FA holds bachelor degree in Business Informatics Engineering at Al-Wadee International University, Master degree in Informatics and Decision Supporting Systems.

MY is a Ph.D. in Computer and Automation Systems, University of Damascus, Syria

Endnote

a. metaheuristic is a higher-level procedure or heuristic designed to find, generate, or select a heuristic (partial search algorithm) that may provide a sufficiently good solution to an optimization problem, especially with incomplete or imperfect information or limited computation capacity.

\section{References}

1. Goodfellow, I., Pouget-Abadie, J., Mirza, M., Xu, B., Warde-Farley, D., Ozair, S., Courville, A., Bengio, Y.: Generative adversarial nets. In: Advances in Neural Information Processing Systems, pp. 2672-2680 (2014)

2. Claesen, M., De Moor, B.: Hyperparameter search in machine learning. arXiv preprint arXiv:1502.02127 (2015)

3. Zhang, H., Sindagi, V., Patel, V.M.: Image de-raining using a conditional generative adversarial network. IEEE transactions on circuits and systems for video technology (2019)

4. Shi, H., Dong, J., Wang, W., Qian, Y., Zhang, X.: Ssgan: secure steganography based on generative adversarial networks. In: Pacific Rim Conference on Multimedia, pp. 534-544 (2017). Springer

5. Shrivastava, A., Pfister, T., Tuzel, O., Susskind, J., Wang, W., Webb, R.: Learning from simulated and unsupervised images through adversarial training. In: Proceedings of the IEEE Conference on Computer Vision and Pattern Recognition, pp. 2107-2116 (2017)

6. Wu, J., Zhang, C., Xue, T., Freeman, B., Tenenbaum, J.: Learning a probabilistic latent space of object shapes via 3d generative-adversarial modeling. In: Advances in Neural Information Processing Systems, pp. 82-90 (2016)

7. Du, L., Cui, Z., Wang, L., Ma, J.: Structure tuning method on deep convolutional generative adversarial network with nondominated sorting genetic algorithm ii. Concurrency and Computation: Practice and Experience, 5688 (2020)

8. Koshiyama, A., Firoozye, N., Treleaven, P.: Generative adversarial networks for financial trading strategies fine-tuning and combination. Quantitative Finance, 1-17 (2020)

9. Douzas, G., Bacao, F.: Effective data generation for imbalanced learning using conditional generative adversarial networks. Expert Systems with applications 91, 464-471 (2018)

10. Chen, T., Lucic, M., Houlsby, N., Gelly, S.: On self modulation for generative adversarial networks. arXiv preprint arXiv: 1810.01365 (2018)

11. Mitchell, M.: An Introduction to Genetic Algorithms. MIT press, ??? (1998)

12. Kramer, O.: Genetic Algorithm Essentials vol. 679. Springer, ??? (2017)

13. Karpathy, A.: Generative Models. OpenAl (2020). https://openai.com/blog/generative-models/

14. Kingma, D.P., Ba, J.: Adam: A method for stochastic optimization. arXiv preprint arXiv:1412.6980 (2014)

15. Gal, Y., Ghahramani, Z.: Dropout as a bayesian approximation: Representing model uncertainty in deep learning. In: International Conference on Machine Learning, pp. 1050-1059 (2016)

16. geneticalgorithm. https://pypi.org/project/geneticalgorithm/

17. Cheng, K., Tahir, R., Eric, L.K., Li, M.: An analysis of generative adversarial networks and variants for image synthesis on mnist dataset. Multimedia Tools and Applications, 1-28 (2020)

\section{Figures}

Figure 1 . Simple GAN

Figure 2 . hyper-parameters vs default parameters

Figure 3 . Generator vs Discriminator

Figure 4 . Python version

Figure 5 . Generator design 
Figure 6 . Generator details

Figure 7 . Discriminator details

Figure 8 . Dataset to Model stages.

Figure 9 . Results for Discriminator Loss minimization.

Figure 10 . Top results of Discriminator test.

Figure 11 . Results for Generator Loss minimization.

Figure 12 . Top results of Generator test.

Figure 13 . Generator results of final testing.

Table 1 Implementation of GAN with GA

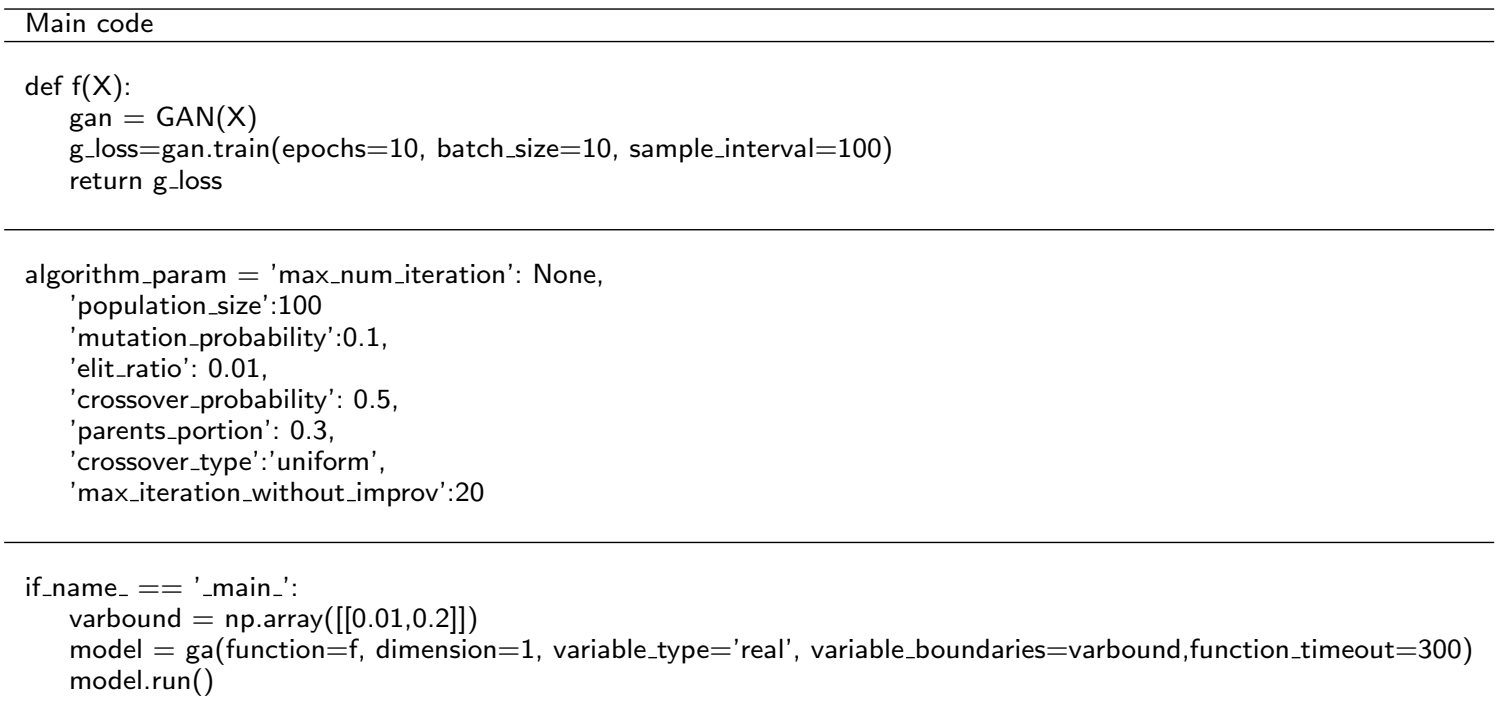

Table 2 Best parameters for minimization Discriminator loss

\begin{tabular}{ccc}
\hline keep probability & Batch normalization & Neurons \\
\hline 0.812 & 64 & $\begin{array}{c}\text { Generator: 256,512,1024 } \\
\text { Discriminator: } 512,256\end{array}$ \\
\hline Learning Rate: 0.00019948 & &
\end{tabular}

Tables

\section{Additional Files}

Additional file 1 - Figure 1.docx

Additional file 2 - Figure 2.docx

Additional file 3 - Figure 3.docx

Additional file 4 - Figure 4.docx

Additional file 5 - Figure 5.docx

Additional file 6 - Figure 6.docx

Additional file 7 - Figure 7.docx

Additional file 8 - Figure 8.docx

Additional file 9 - Figure 9.docx

Additional file 10 - Figure 10.docx 
Table 3 Best parameters for minimization Generator loss

\begin{tabular}{ccc}
\hline keep probability & Batch normalization & Neurons \\
\hline 0.63 & 64 & $\begin{array}{c}\text { Generator: 256,512,1024 } \\
\text { Discriminator: 512,256 }\end{array}$ \\
\hline
\end{tabular}




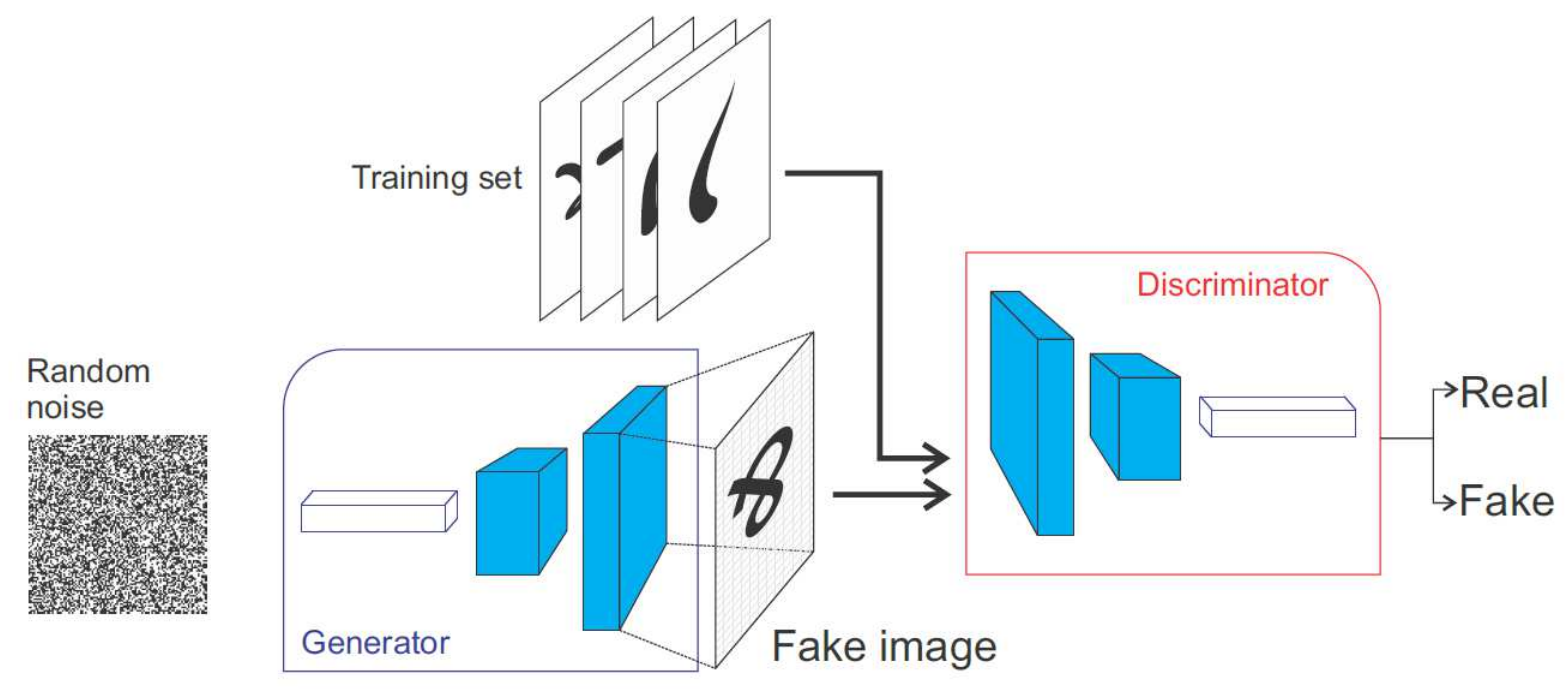




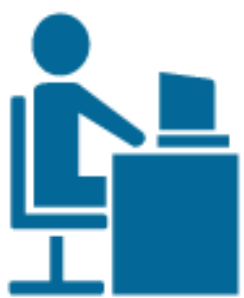

Hyperparameters

O. n_layers $=3$ learning_rate $=0.1$

n_layers $=3$

Q. n_neurons $=1024$ learning_rate $=0.01$

n_layers $=5$

D. n_neurons $=256$ learning rate $=0.1$

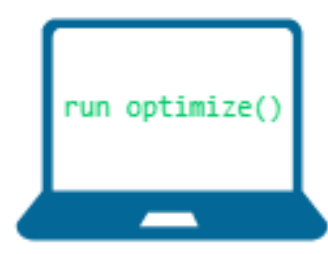

Parameters
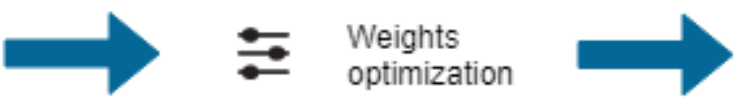

$\because \quad \because \quad \begin{aligned} & \text { Weights } \\ & \text { optimization }\end{aligned}$

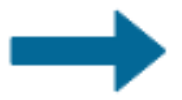

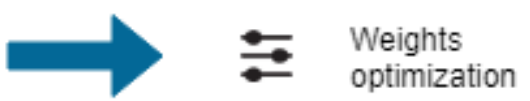

$85 \%$

$80 \%$

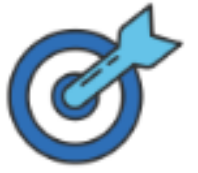

Score

$92 \%$ 


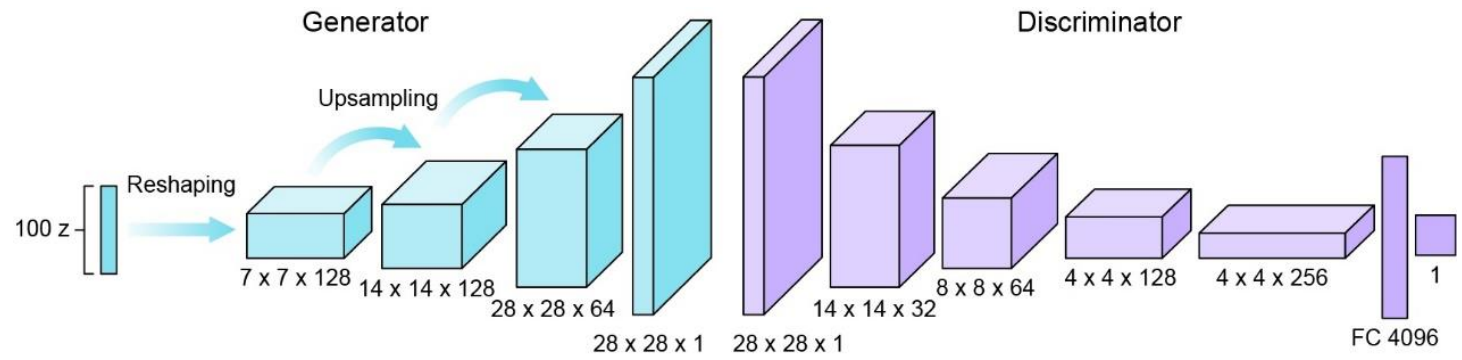


Anaconda Prompt (Anaconda3)

(base) $\mathrm{C}$ : Ussers \user >activate tensorflow

(tensorflow) C: \Users \user >python --version

Python 3.7.4

(tensorflow) C: UUsers \user >pip show tensorflow

Name: tensorflow

Version: 1.14.0

Summary: Tensorflow is an open source machine learning framework for everyone.

Home-page: https://WWw.tensorflow.org/

Author: Google Inc.

Author-email: packages@tensorflow.org

License: Apache 2.0

Location: c: \users \user \anaconda3\envs\tensorflow $\backslash l i b \backslash$ site-packages

Requires: grpcio, wheel, tensorboard, wrapt, tensorflow-estimator, keras-preprocessing, numpy, gast, google-pasta,

absl-py, astor, termcolor, protobuf, keras-applications, six

Required-by:

(tensorflow) C: UUsers \user>o 


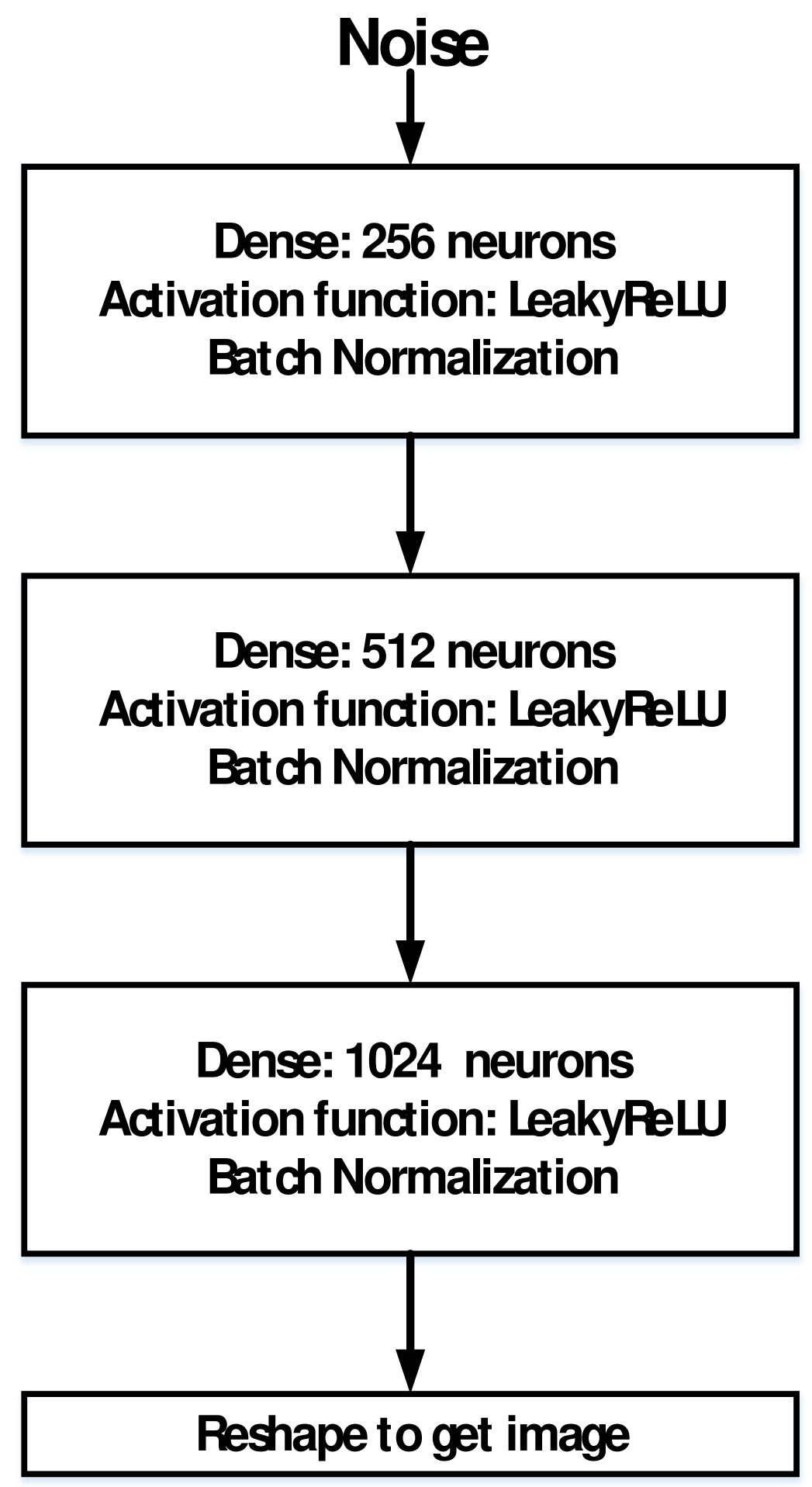




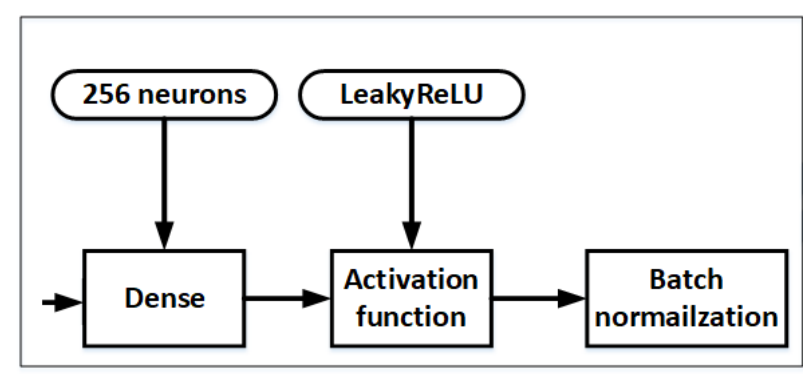

Dense: 256 neurons

Activation function: LeakyReLU

Batch Normalization 


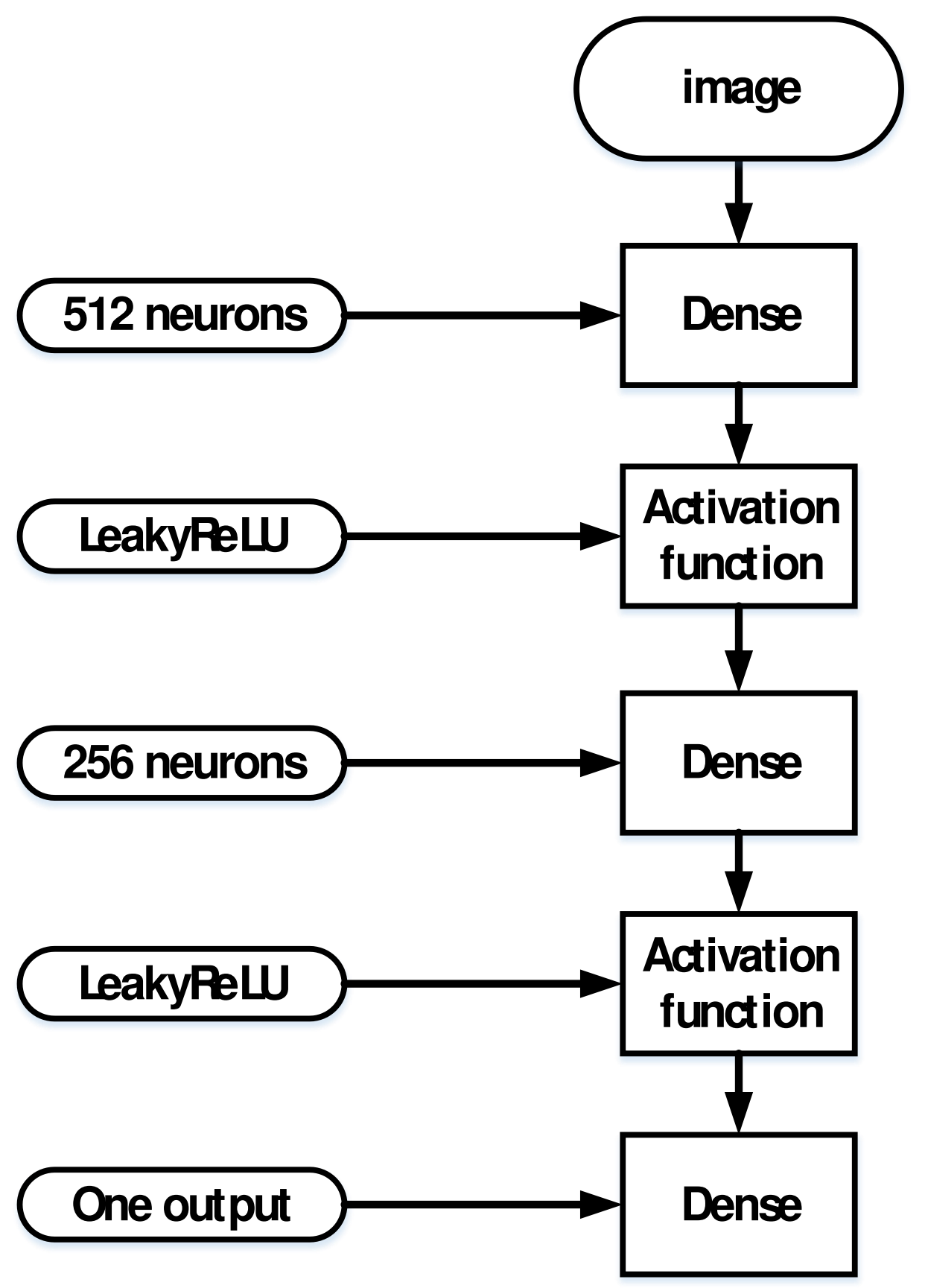




\begin{tabular}{|c|c|c|c|c|}
\hline 1 & G_Loss, & D_loss, & D_ACC, & LR \\
\hline 2 & 4.835429, & 8.059048, & 0.500000, & 0.043611 \\
\hline 3 & 9.805188, & 9.653286 , & 0.400000, & 0.043611 \\
\hline 4 & 8.059048, & 10.450405 , & 0.350000, & 0.043611 \\
\hline 5 & 6.160692, & 13.638883, & 0.150000, & 0.043611 \\
\hline 6 & 9.670857 , & 12.044644, & 0.250000, & 0.043611 \\
\hline 7 & 11.282667 , & 11.247524, & 0.300000, & 0.043611 \\
\hline 8 & 9.670857 & 11.247524, & 0.300000 & 0.043611 \\
\hline 9 & 12.894476, & 11.247524, & 0.300000, & 0.043611 \\
\hline 10 & 9.670857 , & 11.247524, & 0.300000, & 0.043611 \\
\hline 11 & 11.282667 , & 11.247524, & 0.300000, & 0.028553 \\
\hline 12 & 9.670857 , & 10.450405 , & 0.350000, & 0.028553 \\
\hline 13 & 11.282667 , & 11.247524, & 0.300000, & 0.028553 \\
\hline 14 & 11.282667, & 11.247524, & 0.300000, & 0.028553 \\
\hline 15 & 9.670857 & 8.856167, & 0.450000, & 0.028553 \\
\hline 16 & 9.670857 , & 10.450405 , & 0.350000, & 0.028553 \\
\hline 17 & 11.282667 , & 10.450405 , & 0.350000, & 0.028553 \\
\hline 18 & 11.282667 , & 8.059048, & 0.500000, & 0.028553 \\
\hline 19 & 9.670857 , & 10.450405, & 0.350000, & 0.028553 \\
\hline 20 & 8.059048, & 10.450405 , & 0.350000, & 0.028553 \\
\hline 21 & 8.059048, & 11.247524, & 0.300000, & 0.097347 \\
\hline 22 & 9.670857 , & 10.450405 , & 0.350000, & 0.097347 \\
\hline 23 & 6.447238, & 10.450405, & 0.350000, & 0.097347 \\
\hline 24 & 9.670857 , & 8.856167, & 0.450000, & 0.097347 \\
\hline 25 & 8.059048, & 11.247524, & 0.300000, & 0.097347 \\
\hline 26 & 6.447238, & 10.450405 , & 0.350000, & 0.097347 \\
\hline 27 & 12.894476, & 12.044643, & 0.250000, & 0.097347 \\
\hline 28 & 11.282667 , & 11.247524, & 0.300000, & 0.097347 \\
\hline 29 & 12.894476, & 10.450405, & 0.350000 & 0.097347 \\
\hline 30 & 11.282667 , & 10.450405 , & 0.350000, & 0.097347 \\
\hline 31 & 11.282667 , & 11.247524, & 0.300000, & 0.065461 \\
\hline 32 & 8.059048, & 9.653286, & 0.400000, & 0.065461 \\
\hline
\end{tabular}




\begin{tabular}{|c|c|c|c|c|}
\hline & G_Loss & D_loss & ACC & LR \\
\hline 698 & $11.2 \overline{8} 2667$ & $\theta .0$ & $1 . \theta$ & 0.065273 \\
\hline 720 & 9.670857 & $\theta . \theta$ & $1 . \theta$ & 0.066043 \\
\hline 750 & 11.282667 & $\theta . \theta$ & $1 . \theta$ & 0.028301 \\
\hline 824 & 11.282667 & $\theta . \theta$ & $1 . \theta$ & 0.090876 \\
\hline 874 & 12.894476 & $\theta . \theta$ & 1.0 & 0.013635 \\
\hline 881 & 8.059048 & $\theta . \theta$ & $1 . \theta$ & 0.026079 \\
\hline 898 & 11.282667 & $\theta . \theta$ & $1 . \theta$ & 0.020439 \\
\hline 899 & 11.282667 & $\theta . \theta$ & $1 . \theta$ & 0.036774 \\
\hline 943 & 11.282667 & $\theta . \theta$ & $1 . \theta$ & 0.028957 \\
\hline 952 & 12.894476 & $\theta . \theta$ & $1 . \theta$ & 0.044514 \\
\hline 971 & 11.282667 & $\theta . \theta$ & $1 . \theta$ & 0.088288 \\
\hline 986 & 11.282667 & $\theta . \theta$ & $1 . \theta$ & 0.072956 \\
\hline 995 & 11.282667 & $\theta . \theta$ & $1 . \theta$ & 0.067904 \\
\hline 1006 & 12.894476 & $\theta . \theta$ & $1 . \theta$ & 0.019948 \\
\hline 1040 & 9.670857 & $\theta . \theta$ & $1 . \theta$ & 0.034972 \\
\hline 1071 & 11.282667 & $\theta . \theta$ & 1.0 & 0.013635 \\
\hline 1095 & 9.670857 & $\theta .0$ & $1 . \theta$ & 0.067284 \\
\hline 1113 & 11.282667 & $\theta . \theta$ & $1 . \theta$ & 0.034972 \\
\hline 1122 & 11.282667 & $\theta . \theta$ & $1 . \theta$ & 0.067284 \\
\hline 1166 & 9.670857 & $\theta . \theta$ & $1 . \theta$ & 0.068281 \\
\hline 1182 & 11.282667 & $\theta . \theta$ & $1 . \theta$ & 0.034972 \\
\hline 1219 & 11.282667 & $\theta . \theta$ & $1 . \theta$ & 0.034972 \\
\hline 1229 & 8.059048 & $\theta . \theta$ & $1 . \theta$ & 0.090502 \\
\hline 1247 & 11.282667 & $\theta . \theta$ & $1 . \theta$ & 0.085555 \\
\hline 1337 & 11.282667 & $\theta . \theta$ & $1 . \theta$ & 0.064713 \\
\hline 1388 & 11.282667 & $\theta . \theta$ & $1 . \theta$ & 0.032499 \\
\hline 1406 & 11.282667 & $\theta . \theta$ & $1 . \theta$ & 0.090502 \\
\hline 1407 & 9.670857 & $\theta . \theta$ & $1 . \theta$ & 0.090502 \\
\hline 1465 & 12.894476 & $\theta . \theta$ & $1 . \theta$ & 0.052773 \\
\hline 1511 & 11.282667 & $\theta . \theta$ & $1 . \theta$ & 0.085555 \\
\hline $\begin{array}{l}\cdots \\
2277\end{array}$ & 12.894476 & $\ddot{\theta} \dot{\theta}$ & $\ddot{1 . \theta}$ & 0.013635 \\
\hline 2313 & 11.282667 & $\theta . \theta$ & $1 . \theta$ & 0.090502 \\
\hline 2333 & 9.670857 & $\theta . \theta$ & $1 . \theta$ & 0.085555 \\
\hline 2339 & 9.670857 & $\theta . \theta$ & $1 . \theta$ & 0.083484 \\
\hline 2340 & 11.282667 & $\theta . \theta$ & $1 . \theta$ & 0.083484 \\
\hline 2386 & 11.282667 & $\theta . \theta$ & $1 . \theta$ & 0.019948 \\
\hline 2404 & 9.670857 & $\theta . \theta$ & 1.0 & 0.019948 \\
\hline 2438 & 11.282667 & $\theta . \theta$ & $1 . \theta$ & 0.090502 \\
\hline 2439 & 11.282667 & $\theta . \theta$ & $1 . \theta$ & 0.019948 \\
\hline 2459 & 11.282667 & $\theta . \theta$ & 1.0 & 0.085555 \\
\hline 2468 & 12.894476 & $\theta .0$ & $1 . \theta$ & 0.085555 \\
\hline 2512 & 11.282667 & 0.0 & 1.0 & 0.044132 \\
\hline
\end{tabular}




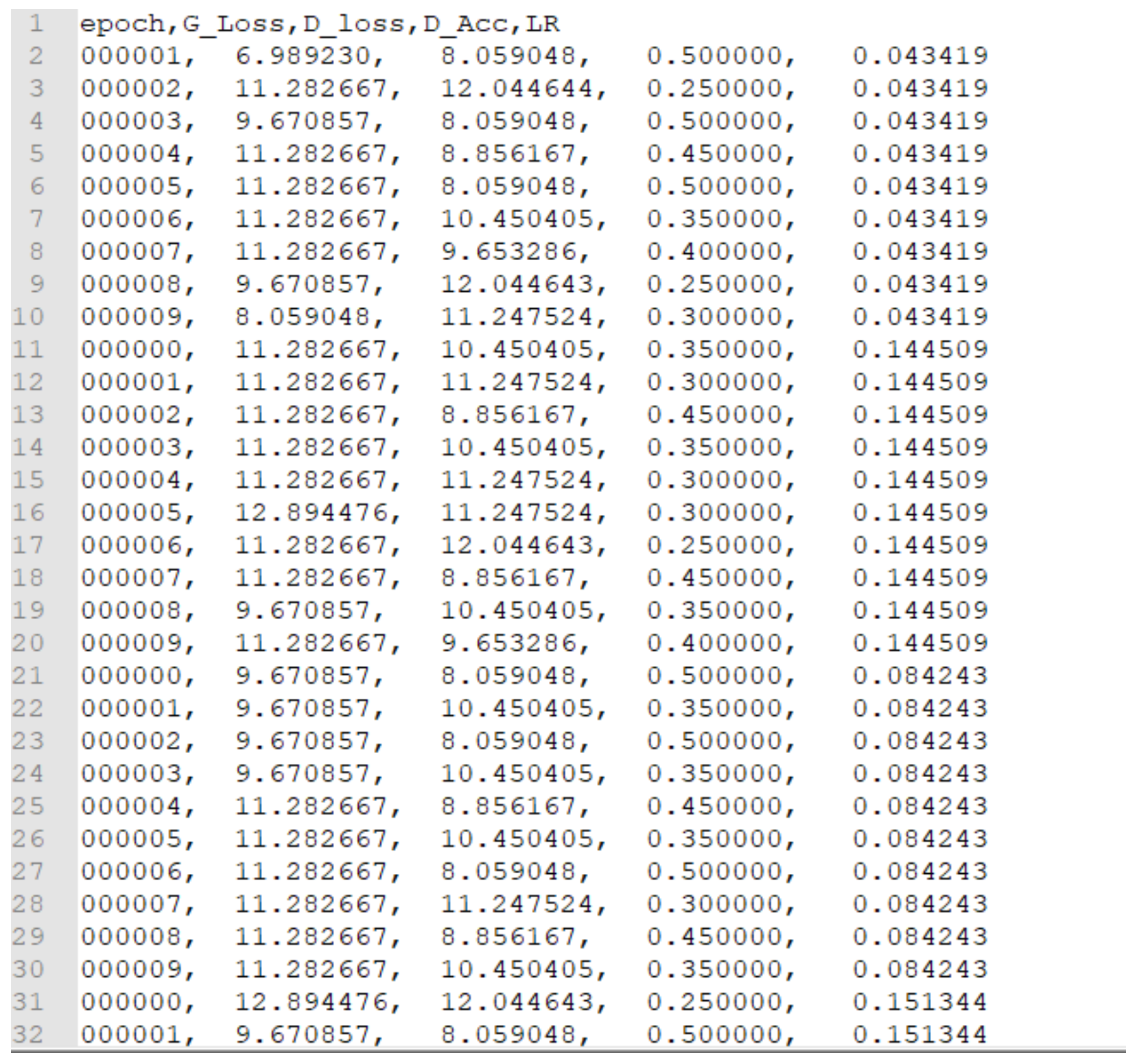




\begin{tabular}{|c|c|c|c|c|c|}
\hline & epoch & G Loss & D 1055 & D $\mathrm{ACC}$ & LR \\
\hline 1333 & 4 & $\theta . \theta$ & $7.1 \overline{7} 4073$ & $\bar{\theta} .55$ & 0.171924 \\
\hline 1334 & 5 & $\theta . \theta$ & 7.971192 & 0.50 & 0.171924 \\
\hline 1338 & 9 & $\theta . \theta$ & 7.971192 & 0.50 & 0.171924 \\
\hline 1339 & $\theta$ & $\theta . \theta$ & 7.971192 & 0.50 & 0.172570 \\
\hline 1340 & 1 & $\theta . \theta$ & 6.376954 & $\theta .60$ & 0.172570 \\
\hline 1344 & 5 & $\theta . \theta$ & 6.376954 & $\theta .60$ & 0.172570 \\
\hline 1346 & 7 & $\theta . \theta$ & 7.174073 & 0.55 & 0.172570 \\
\hline 1349 & $\theta$ & $\theta . \theta$ & 7.174073 & 0.55 & 0.027164 \\
\hline 1350 & 1 & $\theta . \theta$ & 7.174073 & 0.55 & 0.027164 \\
\hline 1351 & 2 & $\theta . \theta$ & 7.971192 & 0.50 & 0.027164 \\
\hline 1353 & 4 & $\theta . \theta$ & 7.971192 & 0.50 & 0.027164 \\
\hline 1359 & $\theta$ & $\theta . \theta$ & 7.174073 & 0.55 & 0.172846 \\
\hline 1360 & 1 & $\theta . \theta$ & 7.174073 & 0.55 & 0.172846 \\
\hline 1362 & 3 & $\theta . \theta$ & 7.174073 & 0.55 & 0.172846 \\
\hline 1365 & 6 & $\theta . \theta$ & 7.971192 & 0.50 & 0.172846 \\
\hline 1366 & 7 & $\theta . \theta$ & 7.971192 & 0.50 & 0.172846 \\
\hline 1367 & 8 & $\theta . \theta$ & 7.971192 & 0.50 & 0.172846 \\
\hline 1368 & 9 & $\theta . \theta$ & 7.174073 & 0.55 & 0.172846 \\
\hline 1371 & 2 & $\theta . \theta$ & 6.376954 & 0.60 & 0.015445 \\
\hline 1373 & 4 & $\theta . \theta$ & 7.971192 & 0.50 & 0.015445 \\
\hline 1376 & 7 & $\theta . \theta$ & 7.174073 & 0.55 & 0.015445 \\
\hline 1378 & 9 & $\theta . \theta$ & 4.782716 & 0.70 & 0.015445 \\
\hline 1379 & $\theta$ & $\theta . \theta$ & 7.971192 & 0.50 & 0.015445 \\
\hline 1381 & 2 & $\theta . \theta$ & 7.971192 & 0.50 & 0.015445 \\
\hline 1382 & 3 & $\theta . \theta$ & 7.971192 & 0.50 & 0.015445 \\
\hline 1384 & 5 & $\theta . \theta$ & 7.971192 & 0.50 & 0.015445 \\
\hline 1385 & 6 & $\theta . \theta$ & 7.174073 & 0.55 & 0.015445 \\
\hline 1386 & 7 & $\theta . \theta$ & 7.174073 & 0.55 & 0.015445 \\
\hline 1389 & $\theta$ & $\theta . \theta$ & 6.376954 & $\theta .60$ & 0.171924 \\
\hline 1390 & 1 & $\theta . \theta$ & 7.971192 & 0.50 & 0.171924 \\
\hline$\ddot{4545}$ & $\cdots$ & $\dot{\theta} \dot{\theta}$ & $\begin{array}{r}\ddot{3} \\
7.971 \dot{192}\end{array}$ & $\ddot{0.50}$ & $0.171 \dot{9} \dot{24}$ \\
\hline 4546 & 7 & $\theta . \theta$ & 7.174073 & 0.55 & 0.171924 \\
\hline 4547 & 8 & $\theta . \theta$ & 7.971192 & 0.50 & 0.171924 \\
\hline 4549 & $\theta$ & $\theta . \theta$ & 7.174073 & 0.55 & 0.171924 \\
\hline 4550 & 1 & $\theta .0$ & 7.971192 & 0.50 & 0.171924 \\
\hline
\end{tabular}




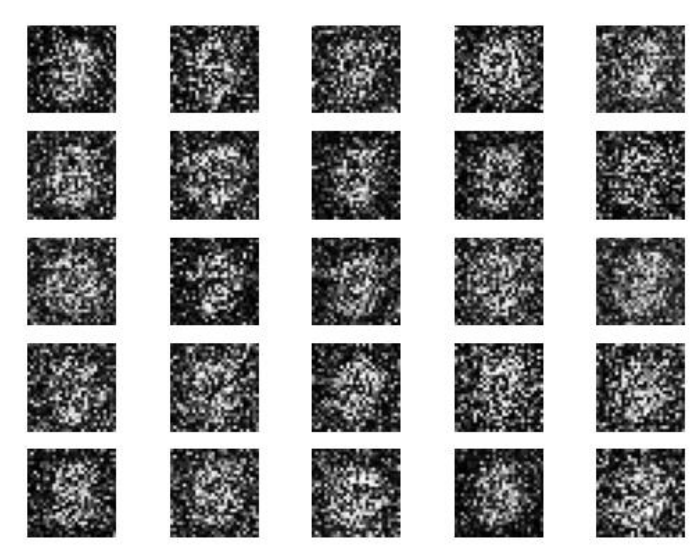

Generator step a

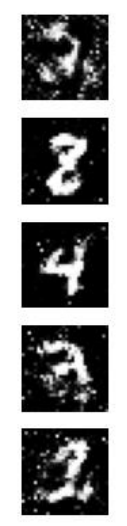

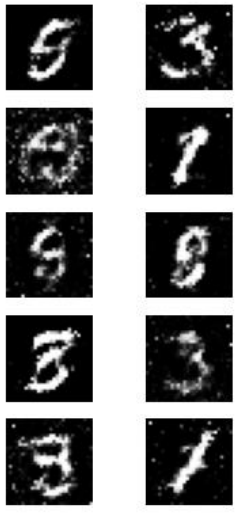

Generator step c

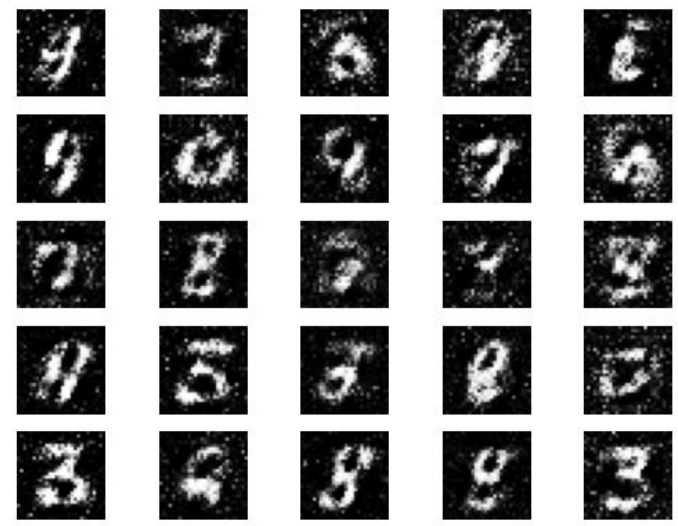

Generator step b

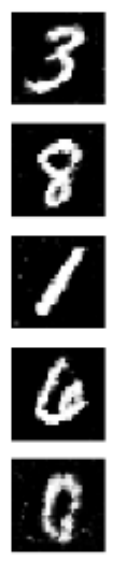

8

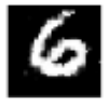

4

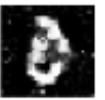

6

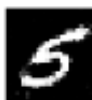

$\$$

w
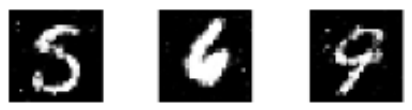

7
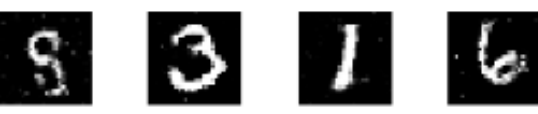

9

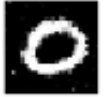

6

1 
Figures

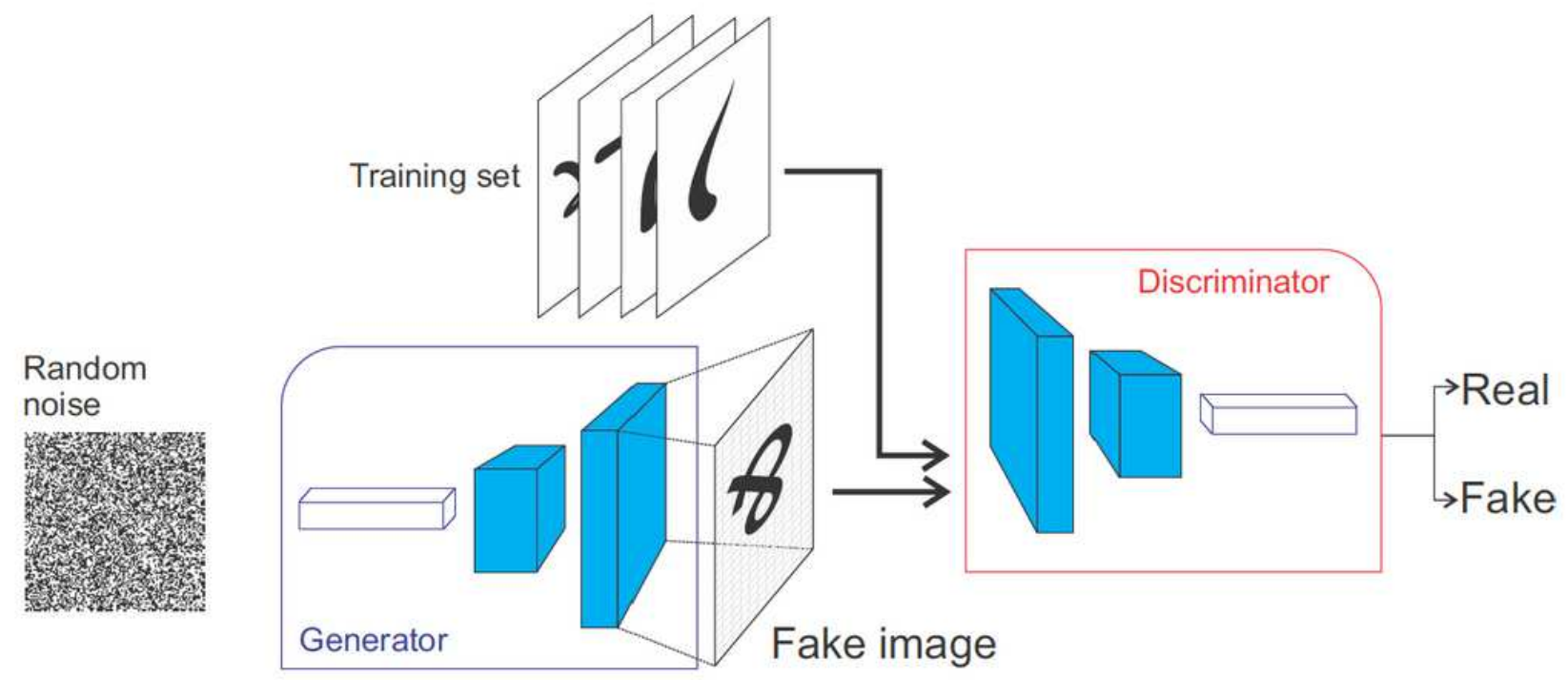

Figure 1

Simple GAN

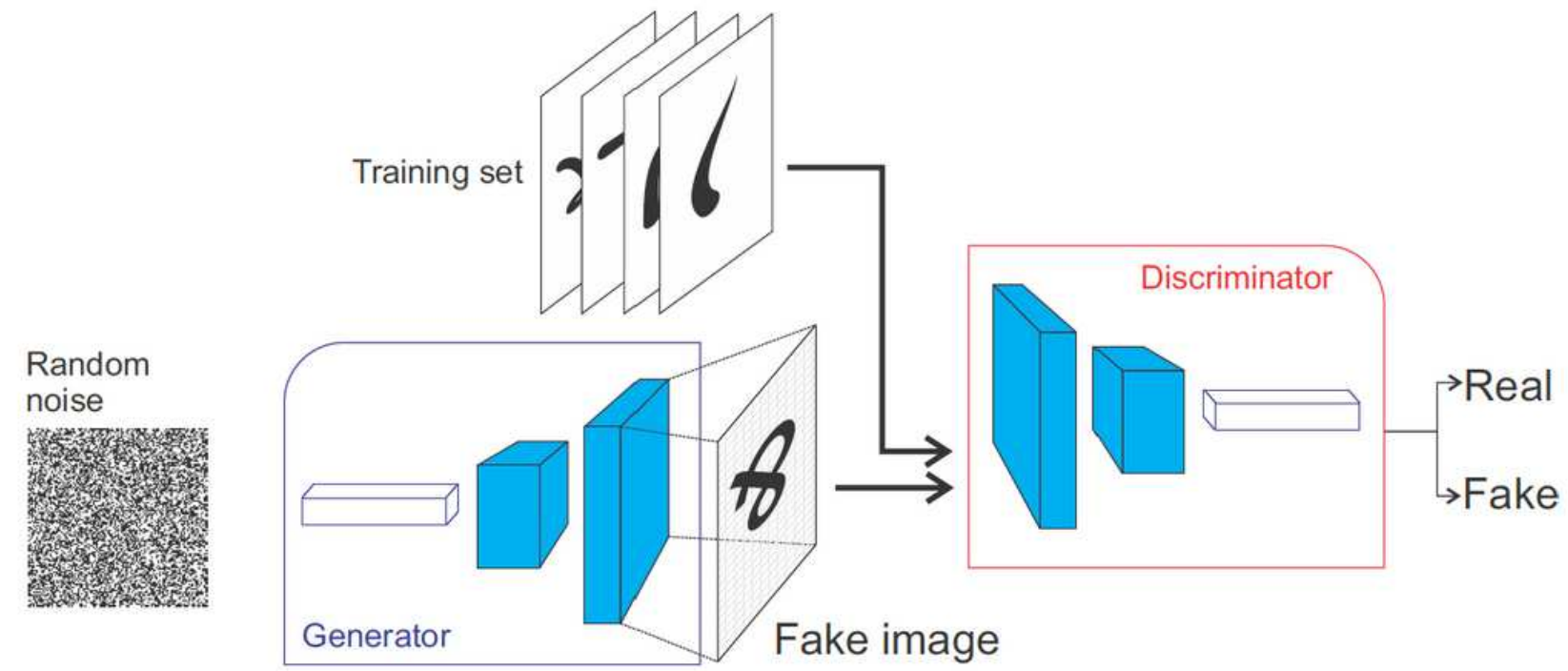

Figure 1

Simple GAN 


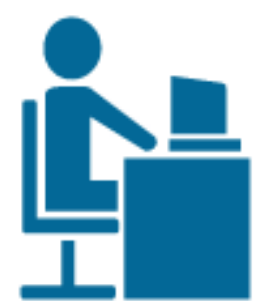

Hyperparameters

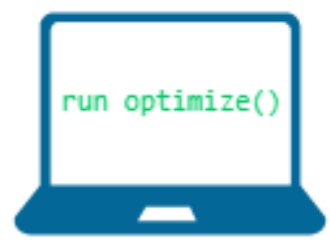

Parameters

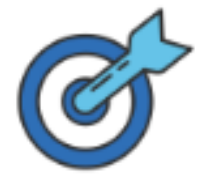

Score

$85 \%$

Weights
optimization

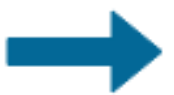

Weights

optimization

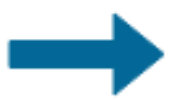

$80 \%$

$92 \%$

\section{Figure 2}

hyper-parameters vs default parameters

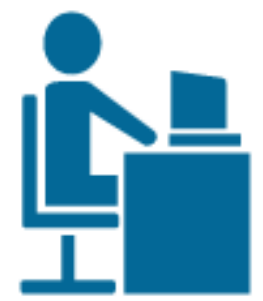

Hyperparameters

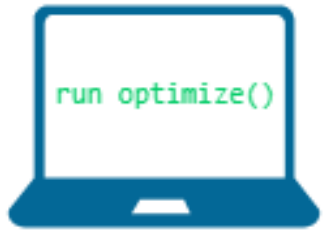

Parameters

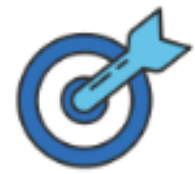

Score

$85 \%$

optimization

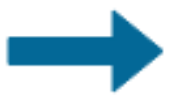

Weights

optimization

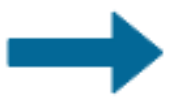

$80 \%$

Weights

optimization

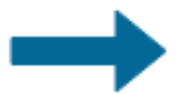

$92 \%$

\section{Figure 2}

hyper-parameters vs default parameters 

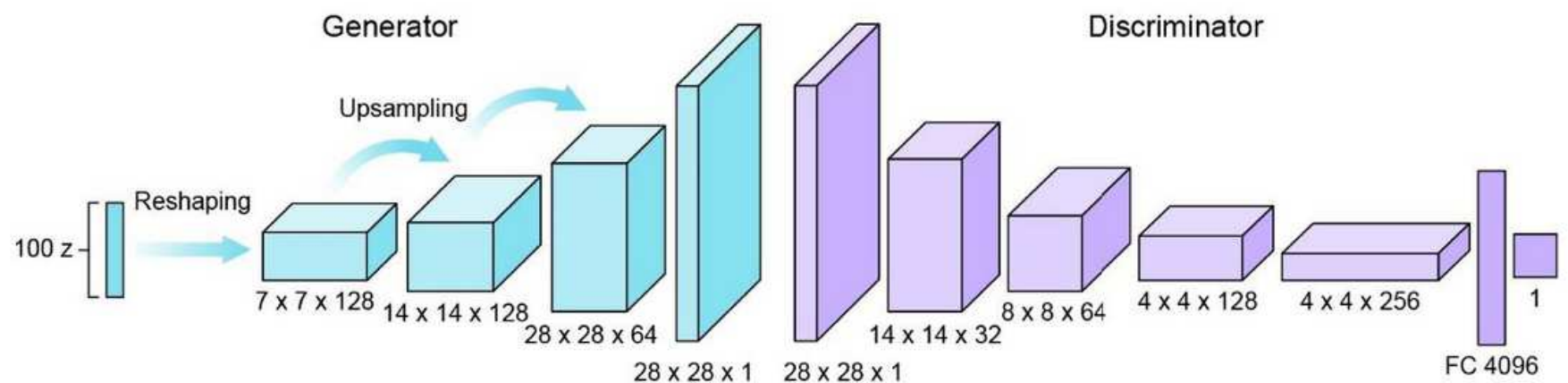

Figure 3

Generator vs Discriminator
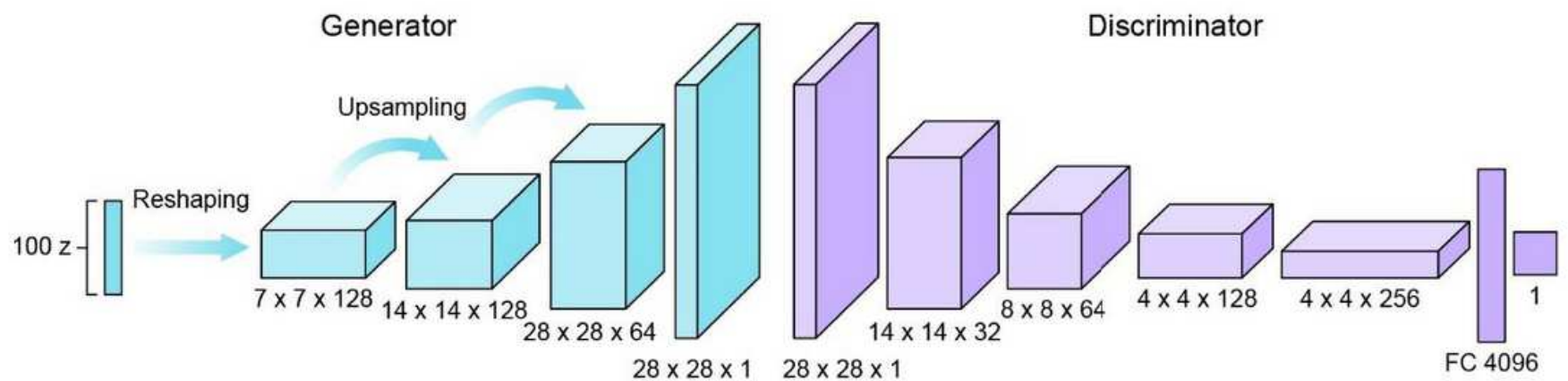

Figure 3

Generator vs Discriminator 
(base) $\mathrm{C}$ : \Users \user>activate tensorflow

(tensorflow) C: UUsers \user>python --version

Python 3.7.4

(tensorflow) $\mathrm{C}$ : \Users \user $>$ pip show tensorflow

Name: tensorflow

Version: 1.14.0

Summary: Tensorflow is an open source machine learning framework for everyone.

Home-page: https://Www.tensorflow.org/

Author: Google Inc.

Author-email: packages@tensorflow.org

License: Apache 2.0

Location: c: \users\user \anaconda3\envs\tensorflow \lib\site-packages

Requires: grpcio, wheel, tensorboard, wrapt, tensorflow-estimator, keras-preprocessing, numpy, gast, google-pasta,

absl-py, astor, termcolor, protobuf, keras-applications, six

Required-by:

(tensorflow) C: UUsers \user>.

\section{Figure 4}

\section{Python version}

Anaconda Prompt (Anaconda3)

$-\square \times$

(base) C: UUsers \user>activate tensorflow

(tensorflow) C: \Users \user>python --version

Python 3.7.4

(tensorflow) C: \Users\user $>$ pip show tensorflow

Name: tensorflow

Version: 1.14 .0

Summary: TensorFlow is an open source machine learning framework for everyone.

Home-page: https://Www.tensorflow.org/

Author: Google Inc.

Author-email: packages@tensorflow.org

License: Apache 2.0

Location: c: \users\user \anaconda3\envs\tensorflow $\backslash 1$ ib \site-packages

Requires: grpcio, wheel, tensorboard, wrapt, tensorflow-estimator, keras-preprocessing, numpy, gast, google-pasta,

absl-py, astor, termcolor, protobuf, keras-applications, six

Required-by:

(tensorflow) C: \Users\user>.

\section{Figure 4}

\section{Python version}




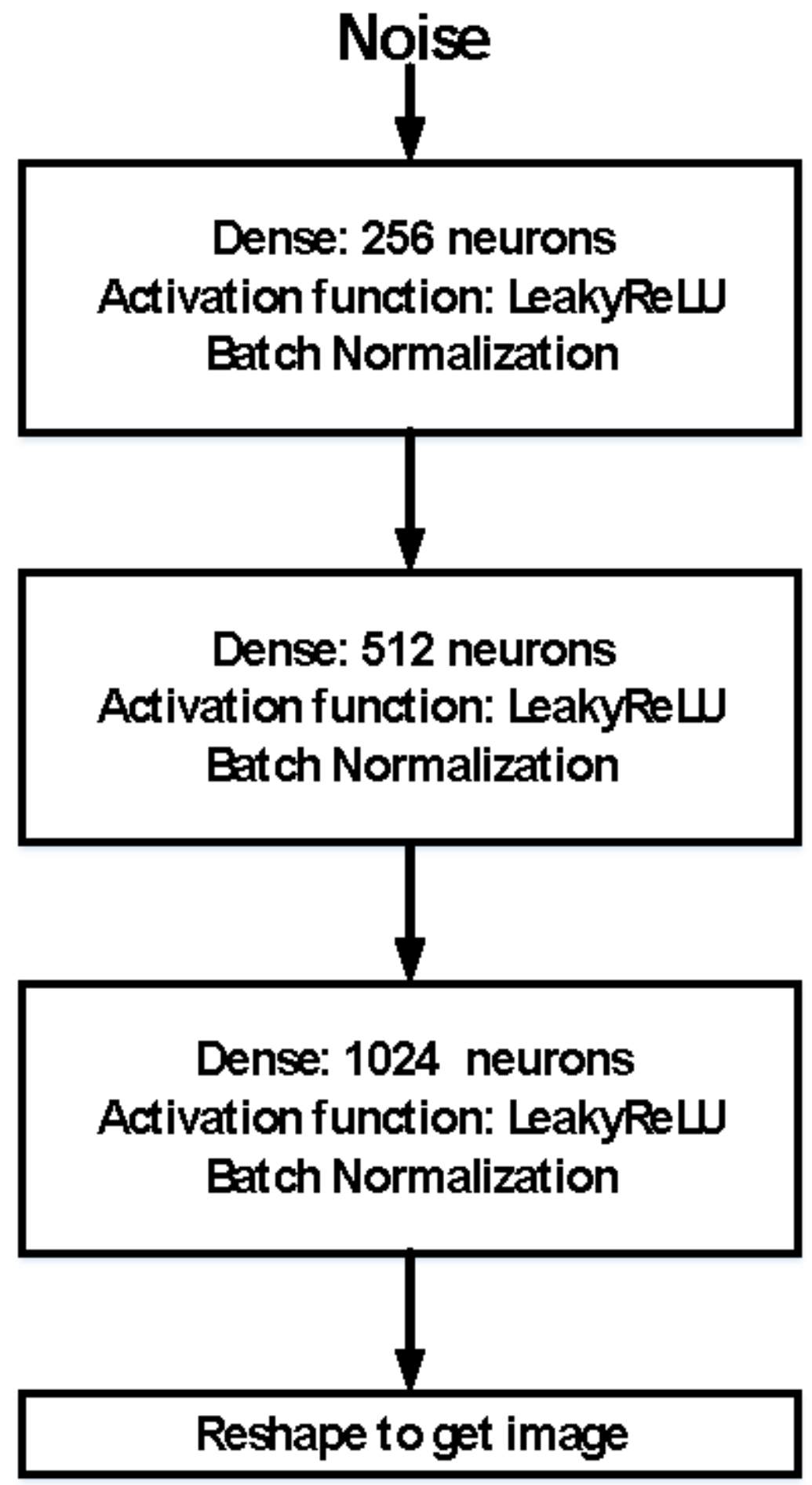

Figure 5

Generator design 


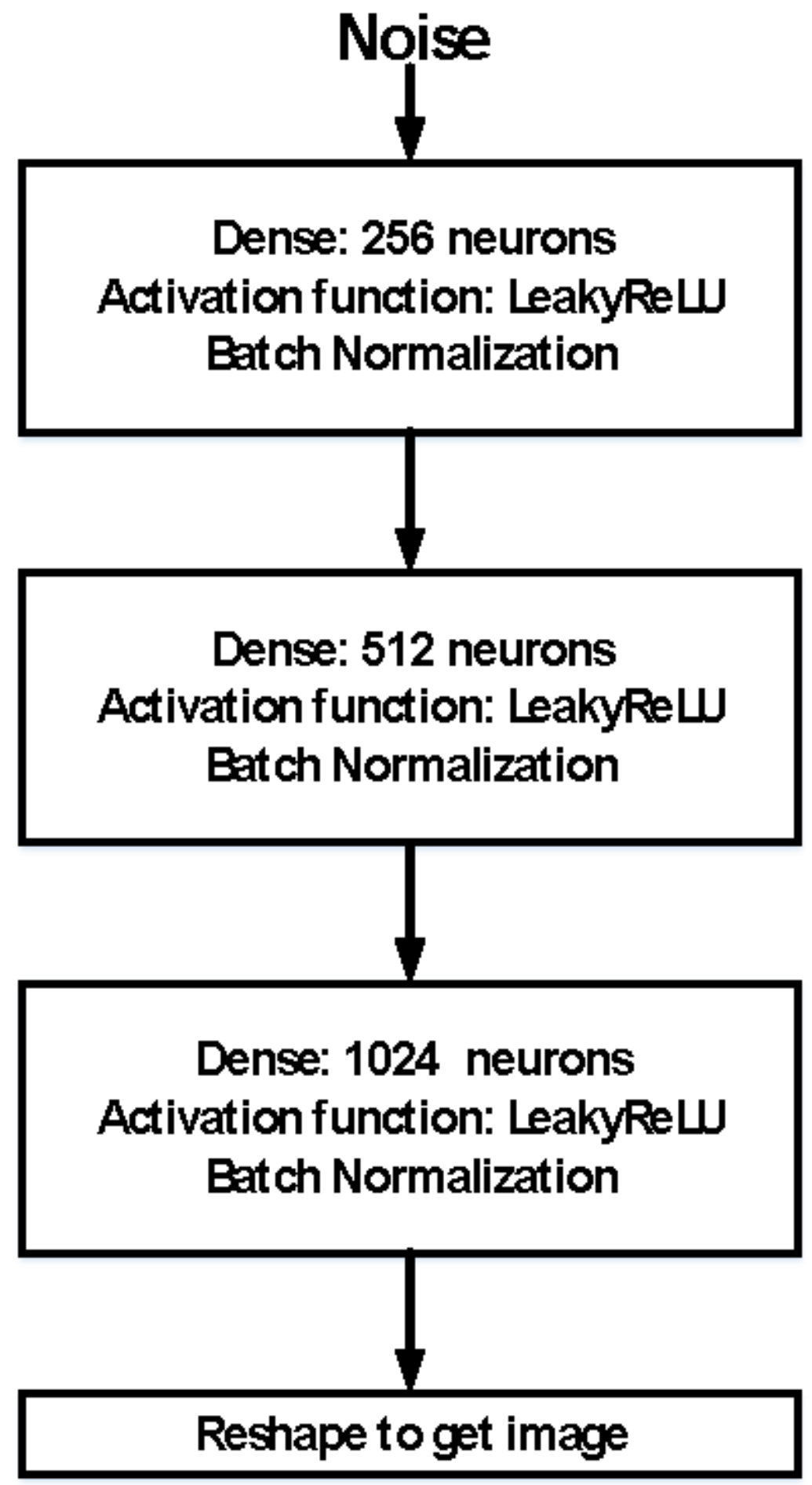

Figure 5

Generator design 


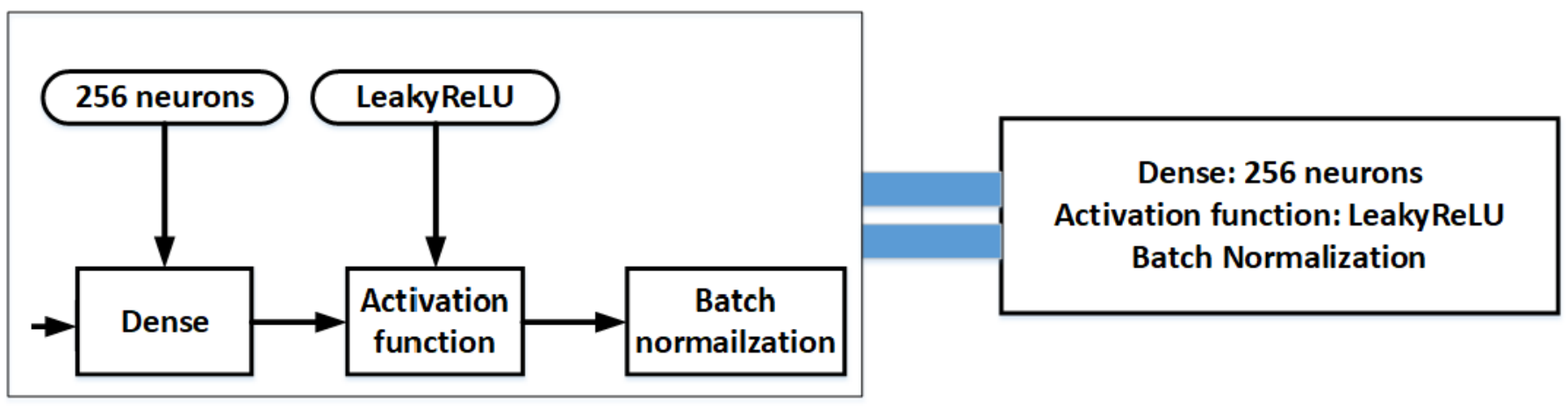

Figure 6

Generator details

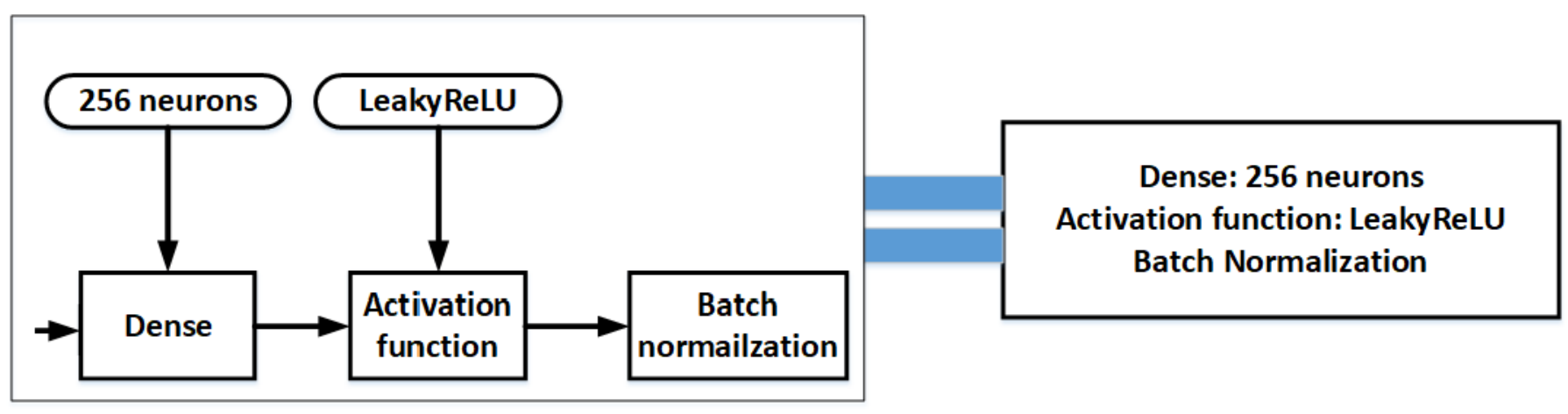

Figure 6

Generator details 


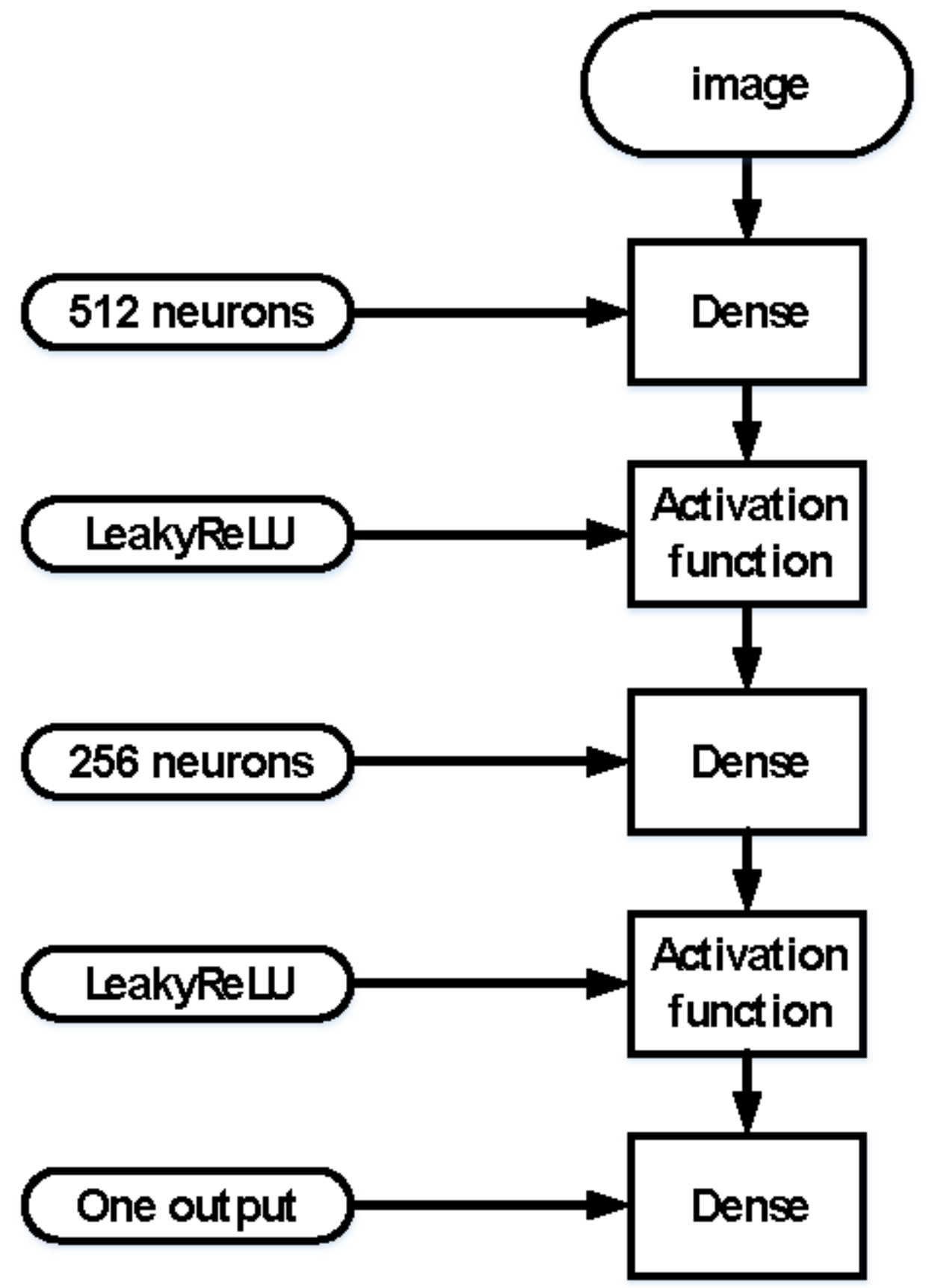

Figure 7

Discriminator details 


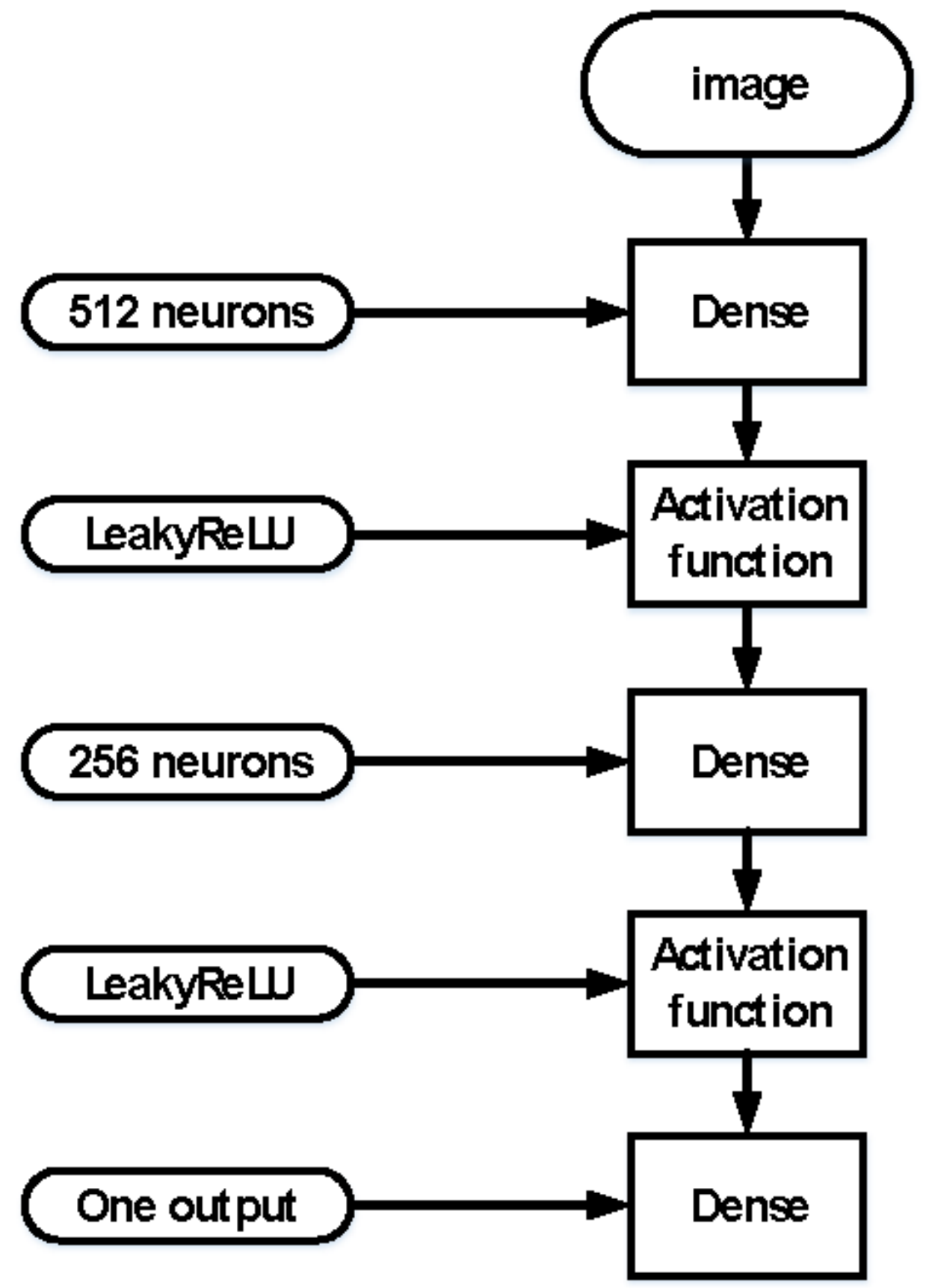

Figure 7

Discriminator details 


\begin{tabular}{|c|c|c|c|c|}
\hline 1 & G_Loss, & D_loss, & D_ACC, & LR \\
\hline 2 & 4.835429, & 8.059048, & 0.500000, & 0.043611 \\
\hline 3 & 9.805188, & 9.653286 , & 0.400000, & 0.043611 \\
\hline 4 & 8.059048, & 10.450405 , & 0.350000, & 0.043611 \\
\hline 5 & 6.160692, & 13.638883, & 0.150000, & 0.043611 \\
\hline 6 & 9.670857 , & 12.044644, & 0.250000, & 0.043611 \\
\hline 7 & 11.282667, & 11.247524, & 0.300000, & 0.043611 \\
\hline 8 & 9.670857 , & 11.247524, & 0.300000, & 0.043611 \\
\hline 9 & 12.894476, & 11.247524, & 0.300000, & 0.043611 \\
\hline 10 & 9.670857 , & 11.247524, & 0.300000, & 0.043611 \\
\hline 11 & 11.282667 , & 11.247524, & 0.300000, & 0.028553 \\
\hline 12 & 9.670857 , & 10.450405 , & 0.350000, & 0.028553 \\
\hline 13 & 11.282667 , & 11.247524, & 0.300000, & 0.028553 \\
\hline 14 & 11.282667 , & 11.247524, & 0.300000, & 0.028553 \\
\hline 15 & 9.670857 , & 8.856167, & 0.450000, & 0.028553 \\
\hline 16 & 9.670857 , & 10.450405 , & 0.350000, & 0.028553 \\
\hline 17 & 11.282667 , & 10.450405, & 0.350000, & 0.028553 \\
\hline 18 & 11.282667 , & 8.059048, & 0.500000 & 0.028553 \\
\hline 19 & 9.670857 , & 10.450405, & 0.350000, & 0.028553 \\
\hline 20 & 8.059048, & 10.450405, & 0.350000, & 0.028553 \\
\hline 21 & 8.059048, & 11.247524, & 0.300000, & 0.097347 \\
\hline 22 & 9.670857 , & 10.450405 , & 0.350000 & 0.097347 \\
\hline 23 & 6.447238, & 10.450405 , & 0.350000, & 0.097347 \\
\hline 24 & 9.670857 , & 8.856167, & 0.450000, & 0.097347 \\
\hline 25 & 8.059048, & 11.247524, & 0.300000, & 0.097347 \\
\hline 26 & 6.447238 & 10.450405 , & 0.350000, & 0.097347 \\
\hline 27 & 12.894476, & 12.044643, & 0.250000, & 0.097347 \\
\hline 28 & 11.282667 , & 11.247524, & 0.300000, & 0.097347 \\
\hline 29 & 12.894476, & 10.450405, & 0.350000, & 0.097347 \\
\hline 30 & 11.282667, & 10.450405 , & 0.350000, & 0.097347 \\
\hline 31 & 11.282667 , & 11.247524, & 0.300000, & 0.065461 \\
\hline 32 & 8.059048, & 9.653286 & 0.400000, & 0.065461 \\
\hline
\end{tabular}

Figure 8

Dataset to Model stages. 


\begin{tabular}{|c|c|c|c|c|}
\hline 1 & G_Loss, & D_loss, & D_ACC, & LR \\
\hline 2 & 4.835429, & 8.059048, & 0.500000, & 0.043611 \\
\hline 3 & 9.805188, & 9.653286 , & 0.400000, & 0.043611 \\
\hline 4 & 8.059048, & 10.450405 , & 0.350000, & 0.043611 \\
\hline 5 & 6.160692, & 13.638883, & 0.150000, & 0.043611 \\
\hline 6 & 9.670857 , & 12.044644, & 0.250000, & 0.043611 \\
\hline 7 & 11.282667, & 11.247524, & 0.300000, & 0.043611 \\
\hline 8 & 9.670857 , & 11.247524, & 0.300000, & 0.043611 \\
\hline 9 & 12.894476, & 11.247524, & 0.300000, & 0.043611 \\
\hline 10 & 9.670857 , & 11.247524, & 0.300000, & 0.043611 \\
\hline 11 & 11.282667 , & 11.247524, & 0.300000, & 0.028553 \\
\hline 12 & 9.670857 , & 10.450405 , & 0.350000, & 0.028553 \\
\hline 13 & 11.282667 , & 11.247524, & 0.300000, & 0.028553 \\
\hline 14 & 11.282667 , & 11.247524, & 0.300000, & 0.028553 \\
\hline 15 & 9.670857 , & 8.856167, & 0.450000, & 0.028553 \\
\hline 16 & 9.670857 , & 10.450405 , & 0.350000, & 0.028553 \\
\hline 17 & 11.282667 , & 10.450405, & 0.350000, & 0.028553 \\
\hline 18 & 11.282667 , & 8.059048, & 0.500000 & 0.028553 \\
\hline 19 & 9.670857 , & 10.450405, & 0.350000, & 0.028553 \\
\hline 20 & 8.059048, & 10.450405, & 0.350000, & 0.028553 \\
\hline 21 & 8.059048, & 11.247524, & 0.300000, & 0.097347 \\
\hline 22 & 9.670857 , & 10.450405 , & 0.350000 & 0.097347 \\
\hline 23 & 6.447238, & 10.450405 , & 0.350000, & 0.097347 \\
\hline 24 & 9.670857 , & 8.856167, & 0.450000, & 0.097347 \\
\hline 25 & 8.059048, & 11.247524, & 0.300000, & 0.097347 \\
\hline 26 & 6.447238 & 10.450405 , & 0.350000, & 0.097347 \\
\hline 27 & 12.894476, & 12.044643, & 0.250000, & 0.097347 \\
\hline 28 & 11.282667 , & 11.247524, & 0.300000, & 0.097347 \\
\hline 29 & 12.894476, & 10.450405, & 0.350000, & 0.097347 \\
\hline 30 & 11.282667, & 10.450405 , & 0.350000, & 0.097347 \\
\hline 31 & 11.282667 , & 11.247524, & 0.300000, & 0.065461 \\
\hline 32 & 8.059048, & 9.653286 & 0.400000, & 0.065461 \\
\hline
\end{tabular}

Figure 8

Dataset to Model stages. 


\begin{tabular}{|c|c|c|c|c|}
\hline & G_Loss & D_loss & D_ACC & LR \\
\hline 698 & $11.2 \overline{8} 2667$ & $\theta .0$ & 1.0 & 0.065273 \\
\hline 720 & 9.670857 & $\theta . \theta$ & $1 . \theta$ & 0.066043 \\
\hline 750 & 11.282667 & $\theta . \theta$ & $1 . \theta$ & 0.028301 \\
\hline 824 & 11.282667 & $\theta . \theta$ & $1 . \theta$ & 0.090876 \\
\hline 874 & 12.894476 & $\theta . \theta$ & 1.0 & 0.013635 \\
\hline 881 & 8.059048 & $\theta .0$ & 1.0 & 0.026079 \\
\hline 898 & 11.282667 & $\theta . \theta$ & 1.0 & 0.020439 \\
\hline 899 & 11.282667 & $\theta . \theta$ & $1 . \theta$ & 0.036774 \\
\hline 943 & 11.282667 & $\theta . \theta$ & $1 . \theta$ & 0.028957 \\
\hline 952 & 12.894476 & $\theta . \theta$ & 1.0 & 0.044514 \\
\hline 971 & 11.282667 & $\theta . \theta$ & 1.0 & 0.088288 \\
\hline 986 & 11.282667 & $\theta . \theta$ & $1 . \theta$ & 0.072956 \\
\hline 995 & 11.282667 & $\theta . \theta$ & $1 . \theta$ & 0.067904 \\
\hline 1006 & 12.894476 & $\theta . \theta$ & 1.0 & 0.019948 \\
\hline 1040 & 9.670857 & $\theta .0$ & $1 . \theta$ & 0.034972 \\
\hline 1071 & 11.282667 & $\theta .0$ & 1.0 & 0.013635 \\
\hline 1095 & 9.670857 & $\theta .0$ & 1.0 & 0.067284 \\
\hline 1113 & 11.282667 & $\theta . \theta$ & $1 . \theta$ & 0.034972 \\
\hline 1122 & 11.282667 & $\theta . \theta$ & 1.0 & 0.067284 \\
\hline 1166 & 9.670857 & $\theta . \theta$ & $1 . \theta$ & 0.068281 \\
\hline 1182 & 11.282667 & $\theta . \theta$ & $1 . \theta$ & 0.034972 \\
\hline 1219 & 11.282667 & $\theta . \theta$ & $1 . \theta$ & 0.034972 \\
\hline 1229 & 8.059048 & $\theta . \theta$ & $1 . \theta$ & 0.090502 \\
\hline 1247 & 11.282667 & $\theta . \theta$ & $1 . \theta$ & 0.085555 \\
\hline 1337 & 11.282667 & $\theta . \theta$ & $1 . \theta$ & 0.064713 \\
\hline 1388 & 11.282667 & $\theta . \theta$ & $1 . \theta$ & 0.032499 \\
\hline 1406 & 11.282667 & $\theta . \theta$ & $1 . \theta$ & 0.090502 \\
\hline 1407 & 9.670857 & $\theta . \theta$ & 1.0 & 0.090502 \\
\hline 1465 & 12.894476 & $\theta .0$ & $1 . \theta$ & 0.052773 \\
\hline 1511 & 11.282667 & $\theta . \theta$ & $1 . \theta$ & 0.085555 \\
\hline $\begin{array}{l}\cdots \\
2277\end{array}$ & 12.894476 & $\ddot{\theta} \dot{\theta}$ & $\ddot{1 . \theta}$ & 0.013635 \\
\hline 2313 & 11.282667 & $\theta .0$ & 1.0 & 0.090502 \\
\hline 2333 & 9.670857 & $\theta . \theta$ & 1.0 & 0.085555 \\
\hline 2339 & 9.670857 & $\theta . \theta$ & $1 . \theta$ & 0.083484 \\
\hline 2340 & 11.282667 & $\theta . \theta$ & $1 . \theta$ & 0.083484 \\
\hline 2386 & 11.282667 & $\theta . \theta$ & $1 . \theta$ & 0.019948 \\
\hline 2404 & 9.670857 & $\theta . \theta$ & 1.0 & 0.019948 \\
\hline 2438 & 11.282667 & $\theta . \theta$ & $1 . \theta$ & 0.090502 \\
\hline 2439 & 11.282667 & $\theta . \theta$ & 1.0 & 0.019948 \\
\hline 2459 & 11.282667 & $\theta . \theta$ & $1 . \theta$ & 0.085555 \\
\hline 2468 & 12.894476 & $\theta . \theta$ & $1 . \theta$ & 0.085555 \\
\hline 2512 & 11.282667 & $\theta . \theta$ & $1 . \theta$ & 0.044132 \\
\hline
\end{tabular}

\section{Figure 9}

Results for Discriminator Loss minimization. 


\begin{tabular}{|c|c|c|c|c|}
\hline & G_Loss & D_10s5 & D_ACC & LR \\
\hline 698 & $11.2 \overline{8} 2667$ & $\theta .0$ & 1.0 & 0.065273 \\
\hline 720 & 9.670857 & $\theta . \theta$ & $1 . \theta$ & 0.066043 \\
\hline 750 & 11.282667 & $\theta . \theta$ & $1 . \theta$ & 0.028301 \\
\hline 824 & 11.282667 & $\theta . \theta$ & $1 . \theta$ & 0.090876 \\
\hline 874 & 12.894476 & $\theta .0$ & 1.0 & 0.013635 \\
\hline 881 & 8.059048 & $\theta . \theta$ & $1 . \theta$ & 0.026079 \\
\hline 898 & 11.282667 & $\theta . \theta$ & $1 . \theta$ & 0.020439 \\
\hline 899 & 11.282667 & $\theta . \theta$ & $1 . \theta$ & 0.036774 \\
\hline 943 & 11.282667 & $\theta . \theta$ & $1 . \theta$ & 0.028957 \\
\hline 952 & 12.894476 & $\theta . \theta$ & $1 . \theta$ & 0.044514 \\
\hline 971 & 11.282667 & $\theta . \theta$ & $1 . \theta$ & 0.088288 \\
\hline 986 & 11.282667 & $\theta . \theta$ & 1.0 & 0.072956 \\
\hline 995 & 11.282667 & $\theta . \theta$ & $1 . \theta$ & 0.067904 \\
\hline 1006 & 12.894476 & $\theta . \theta$ & $1 . \theta$ & 0.019948 \\
\hline 1040 & 9.670857 & $\theta . \theta$ & $1 . \theta$ & 0.034972 \\
\hline 1071 & 11.282667 & $\theta . \theta$ & $1 . \theta$ & 0.013635 \\
\hline 1095 & 9.670857 & $\theta . \theta$ & $1 . \theta$ & 0.067284 \\
\hline 1113 & 11.282667 & $\theta . \theta$ & $1 . \theta$ & 0.034972 \\
\hline 1122 & 11.282667 & $\theta . \theta$ & $1 . \theta$ & 0.067284 \\
\hline 1166 & 9.670857 & $\theta . \theta$ & $1 . \theta$ & 0.068281 \\
\hline 1182 & 11.282667 & $\theta . \theta$ & 1.0 & 0.034972 \\
\hline 1219 & 11.282667 & $\theta . \theta$ & $1 . \theta$ & 0.034972 \\
\hline 1229 & 8.059048 & $\theta . \theta$ & 1.0 & 0.090502 \\
\hline 1247 & 11.282667 & $\theta . \theta$ & 1.0 & 0.085555 \\
\hline 1337 & 11.282667 & $\theta . \theta$ & $1 . \theta$ & 0.064713 \\
\hline 1388 & 11.282667 & $\theta . \theta$ & $1 . \theta$ & 0.032499 \\
\hline 1406 & 11.282667 & $\theta . \theta$ & $1 . \theta$ & 0.090502 \\
\hline 1407 & 9.670857 & $\theta . \theta$ & 1.0 & 0.090502 \\
\hline 1465 & 12.894476 & $\theta . \theta$ & 1.0 & 0.052773 \\
\hline 1511 & 11.282667 & $\theta . \theta$ & $1 . \theta$ & 0.085555 \\
\hline $\begin{array}{l}\cdots \\
2277\end{array}$ & 12.894476 & $\ddot{\theta} \dot{\theta}$ & $\ddot{1} \dot{\theta}$ & 0.013635 \\
\hline 2313 & 11.282667 & $\theta . \theta$ & $1 . \theta$ & 0.090502 \\
\hline 2333 & 9.670857 & $\theta . \theta$ & $1 . \theta$ & 0.085555 \\
\hline 2339 & 9.670857 & $\theta . \theta$ & $1 . \theta$ & 0.083484 \\
\hline 2340 & 11.282667 & $\theta . \theta$ & $1 . \theta$ & 0.083484 \\
\hline 2386 & 11.282667 & $\theta . \theta$ & 1.0 & 0.019948 \\
\hline 2404 & 9.670857 & $\theta . \theta$ & $1 . \theta$ & 0.019948 \\
\hline 2438 & 11.282667 & $\theta . \theta$ & $1 . \theta$ & 0.090502 \\
\hline 2439 & 11.282667 & $\theta .0$ & 1.0 & 0.019948 \\
\hline 2459 & 11.282667 & $\theta . \theta$ & $1 . \theta$ & 0.085555 \\
\hline 2468 & 12.894476 & $\theta . \theta$ & $1 . \theta$ & 0.085555 \\
\hline 2512 & 11.282667 & $\theta . \theta$ & $1 . \theta$ & 0.044132 \\
\hline
\end{tabular}

\section{Figure 9}

Results for Discriminator Loss minimization. 


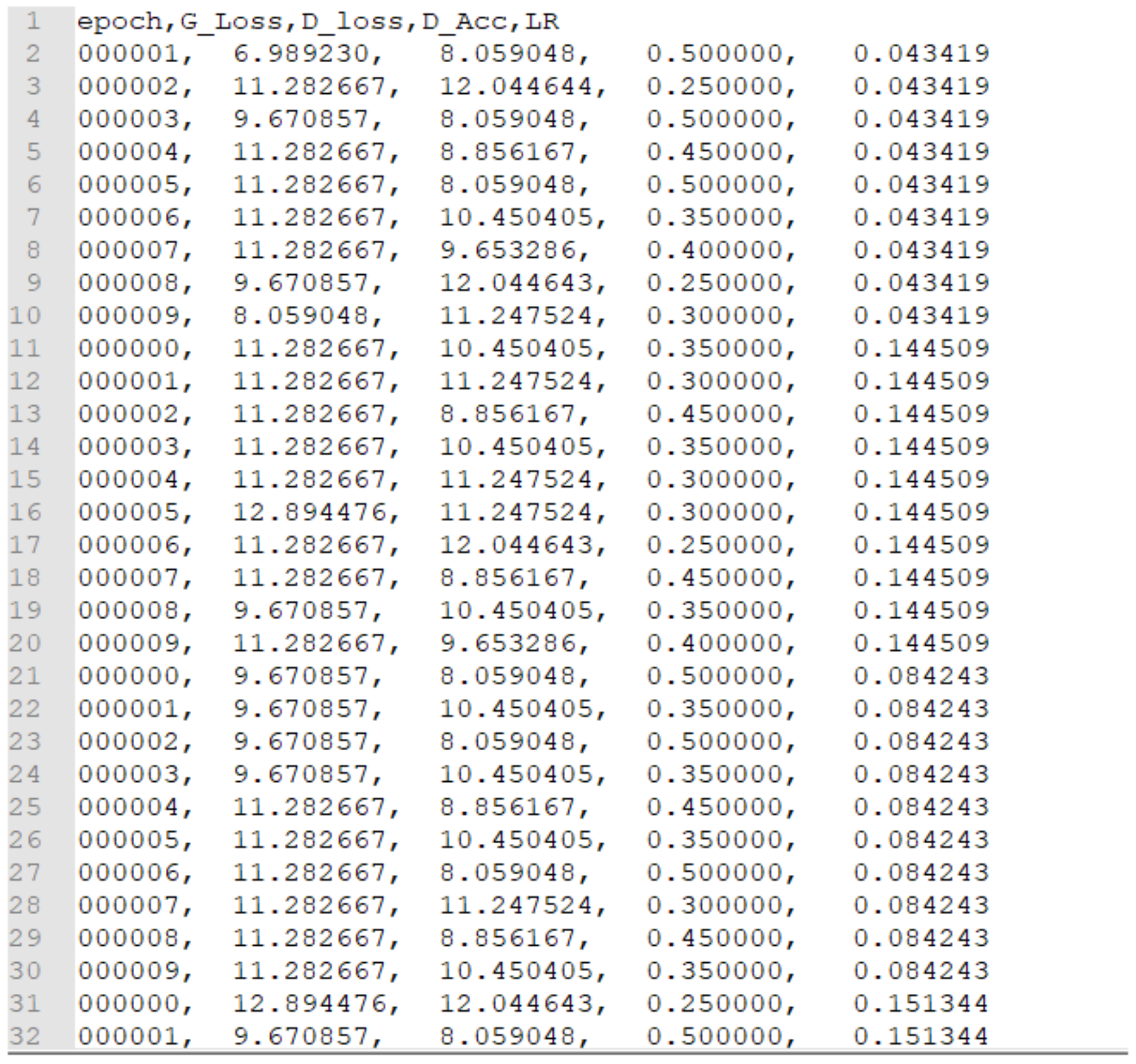

Figure 10

Top results of Discriminator test. 


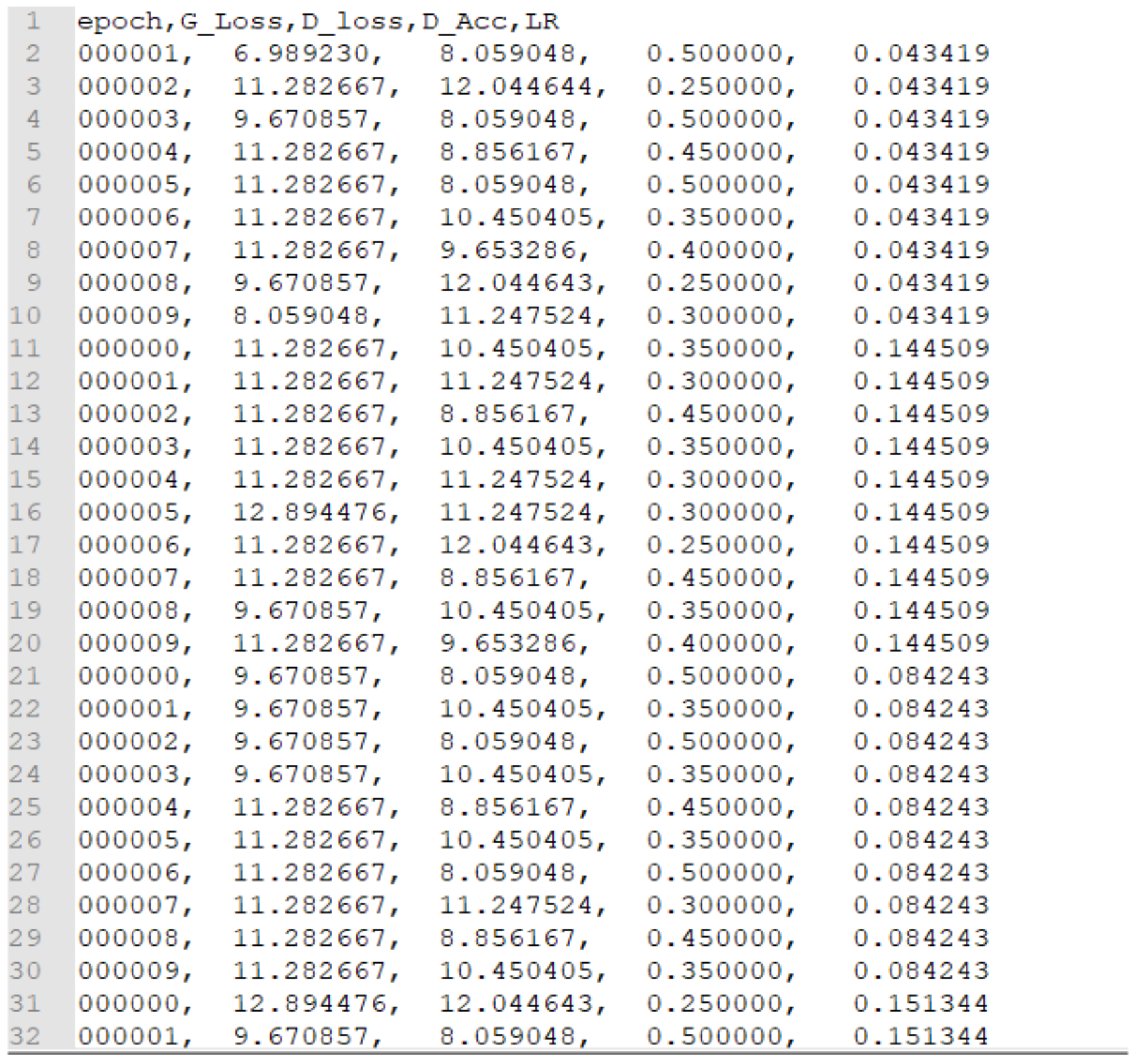

Figure 10

Top results of Discriminator test. 


\begin{tabular}{|c|c|c|c|c|c|}
\hline & epoch & G_Los5 & D_loss & D_ACC & LR \\
\hline 1333 & 4 & $-\theta . \theta$ & $7.1 \overline{7} 4073$ & $\bar{\theta} .55$ & 0.171924 \\
\hline 1334 & 5 & 0.0 & 7.971192 & 0.50 & 0.171924 \\
\hline 1338 & 9 & 0.0 & 7.971192 & 0.50 & 0.171924 \\
\hline 1339 & $\theta$ & 0.0 & 7.971192 & 0.50 & 0.172570 \\
\hline 1340 & 1 & 0.0 & 6.376954 & 0.60 & 0.172570 \\
\hline 1344 & 5 & 0.0 & 6.376954 & 0.60 & 0.172570 \\
\hline 1346 & 7 & 0.0 & 7.174073 & 0.55 & 0.172570 \\
\hline 1349 & $\theta$ & 0.0 & 7.174073 & 0.55 & 0.027164 \\
\hline 1350 & 1 & 0.0 & 7.174073 & 0.55 & 0.027164 \\
\hline 1351 & 2 & 0.0 & 7.971192 & 0.50 & 0.027164 \\
\hline 1353 & 4 & 0.0 & 7.971192 & 0.50 & 0.027164 \\
\hline 1359 & $\theta$ & 0.0 & 7.174073 & 0.55 & 0.172846 \\
\hline 1360 & 1 & 0.0 & 7.174073 & 0.55 & 0.172846 \\
\hline 1362 & 3 & 0.0 & 7.174073 & 0.55 & 0.172846 \\
\hline 1365 & 6 & 0.0 & 7.971192 & 0.50 & 0.172846 \\
\hline 1366 & 7 & 0.0 & 7.971192 & 0.50 & 0.172846 \\
\hline 1367 & 8 & 0.0 & 7.971192 & 0.50 & 0.172846 \\
\hline 1368 & 9 & 0.0 & 7.174073 & 0.55 & 0.172846 \\
\hline 1371 & 2 & 0.0 & 6.376954 & 0.60 & 0.015445 \\
\hline 1373 & 4 & 0.0 & 7.971192 & 0.50 & 0.015445 \\
\hline 1376 & 7 & 0.0 & 7.174073 & 0.55 & 0.015445 \\
\hline 1378 & 9 & 0.0 & 4.782716 & 0.70 & 0.015445 \\
\hline 1379 & $\theta$ & 0.0 & 7.971192 & 0.50 & 0.015445 \\
\hline 1381 & 2 & $\theta .0$ & 7.971192 & 0.50 & 0.015445 \\
\hline 1382 & 3 & 0.0 & 7.971192 & 0.50 & 0.015445 \\
\hline 1384 & 5 & 0.0 & 7.971192 & 0.50 & 0.015445 \\
\hline 1385 & 6 & 0.0 & 7.174073 & 0.55 & 0.015445 \\
\hline 1386 & 7 & 0.0 & 7.174073 & 0.55 & 0.015445 \\
\hline 1389 & $\theta$ & 0.0 & 6.376954 & 0.60 & 0.171924 \\
\hline 1390 & 1 & 0.0 & 7.971192 & 0.50 & 0.171924 \\
\hline$\cdots$ & . & $\cdots$ & $\ldots$ & $\cdots$ & $\ldots$ \\
\hline 4545 & 6 & 0.0 & 7.971192 & 0.50 & 0.171924 \\
\hline 4546 & 7 & 0.0 & 7.174073 & 0.55 & 0.171924 \\
\hline 4547 & 8 & 0.0 & 7.971192 & 0.50 & 0.171924 \\
\hline 4549 & $\theta$ & 0.0 & 7.174073 & 0.55 & 0.171924 \\
\hline 4550 & 1 & 0.0 & 7.971192 & 0.50 & 0.171924 \\
\hline
\end{tabular}

\section{Figure 11}

Results for Generator Loss minimization. 


\begin{tabular}{|c|c|c|c|c|c|}
\hline & epoch & G_Los5 & D_loss & D_ACC & LR \\
\hline 1333 & 4 & $-\theta . \theta$ & $7.1 \overline{7} 4073$ & $\bar{\theta} .55$ & 0.171924 \\
\hline 1334 & 5 & 0.0 & 7.971192 & 0.50 & 0.171924 \\
\hline 1338 & 9 & 0.0 & 7.971192 & 0.50 & 0.171924 \\
\hline 1339 & $\theta$ & 0.0 & 7.971192 & 0.50 & 0.172570 \\
\hline 1340 & 1 & 0.0 & 6.376954 & 0.60 & 0.172570 \\
\hline 1344 & 5 & 0.0 & 6.376954 & 0.60 & 0.172570 \\
\hline 1346 & 7 & 0.0 & 7.174073 & 0.55 & 0.172570 \\
\hline 1349 & $\theta$ & 0.0 & 7.174073 & 0.55 & 0.027164 \\
\hline 1350 & 1 & 0.0 & 7.174073 & 0.55 & 0.027164 \\
\hline 1351 & 2 & 0.0 & 7.971192 & 0.50 & 0.027164 \\
\hline 1353 & 4 & 0.0 & 7.971192 & 0.50 & 0.027164 \\
\hline 1359 & $\theta$ & 0.0 & 7.174073 & 0.55 & 0.172846 \\
\hline 1360 & 1 & 0.0 & 7.174073 & 0.55 & 0.172846 \\
\hline 1362 & 3 & 0.0 & 7.174073 & 0.55 & 0.172846 \\
\hline 1365 & 6 & 0.0 & 7.971192 & 0.50 & 0.172846 \\
\hline 1366 & 7 & 0.0 & 7.971192 & 0.50 & 0.172846 \\
\hline 1367 & 8 & 0.0 & 7.971192 & 0.50 & 0.172846 \\
\hline 1368 & 9 & 0.0 & 7.174073 & 0.55 & 0.172846 \\
\hline 1371 & 2 & 0.0 & 6.376954 & 0.60 & 0.015445 \\
\hline 1373 & 4 & 0.0 & 7.971192 & 0.50 & 0.015445 \\
\hline 1376 & 7 & 0.0 & 7.174073 & 0.55 & 0.015445 \\
\hline 1378 & 9 & 0.0 & 4.782716 & 0.70 & 0.015445 \\
\hline 1379 & $\theta$ & 0.0 & 7.971192 & 0.50 & 0.015445 \\
\hline 1381 & 2 & $\theta .0$ & 7.971192 & 0.50 & 0.015445 \\
\hline 1382 & 3 & 0.0 & 7.971192 & 0.50 & 0.015445 \\
\hline 1384 & 5 & 0.0 & 7.971192 & 0.50 & 0.015445 \\
\hline 1385 & 6 & 0.0 & 7.174073 & 0.55 & 0.015445 \\
\hline 1386 & 7 & 0.0 & 7.174073 & 0.55 & 0.015445 \\
\hline 1389 & $\theta$ & 0.0 & 6.376954 & 0.60 & 0.171924 \\
\hline 1390 & 1 & 0.0 & 7.971192 & 0.50 & 0.171924 \\
\hline$\cdots$ & . & $\cdots$ & $\ldots$ & $\cdots$ & $\ldots$ \\
\hline 4545 & 6 & 0.0 & 7.971192 & 0.50 & 0.171924 \\
\hline 4546 & 7 & 0.0 & 7.174073 & 0.55 & 0.171924 \\
\hline 4547 & 8 & 0.0 & 7.971192 & 0.50 & 0.171924 \\
\hline 4549 & $\theta$ & 0.0 & 7.174073 & 0.55 & 0.171924 \\
\hline 4550 & 1 & 0.0 & 7.971192 & 0.50 & 0.171924 \\
\hline
\end{tabular}

\section{Figure 11}

Results for Generator Loss minimization. 


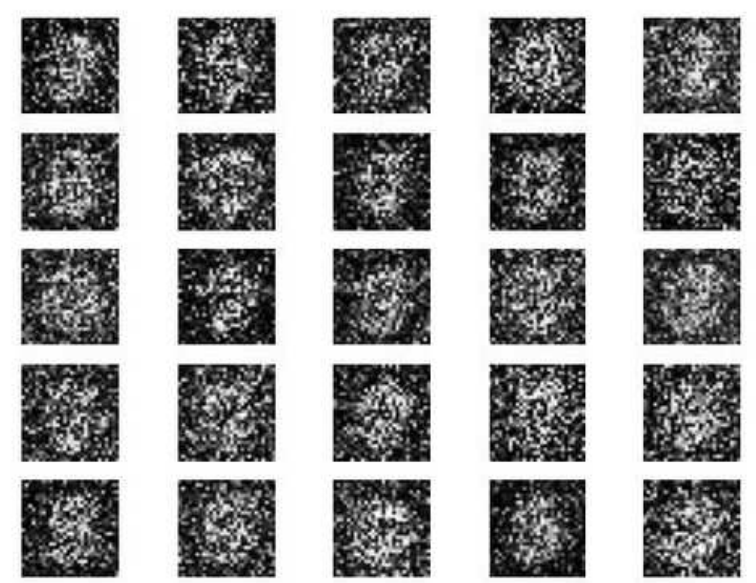

Generator step a
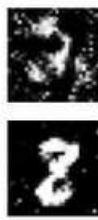

4

a)

1
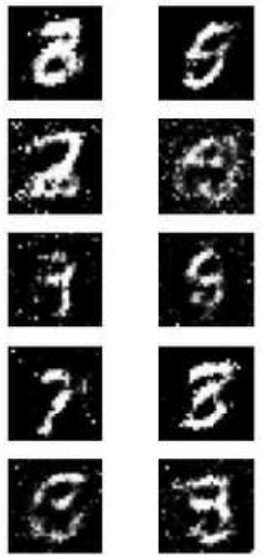

sty

Generator step c
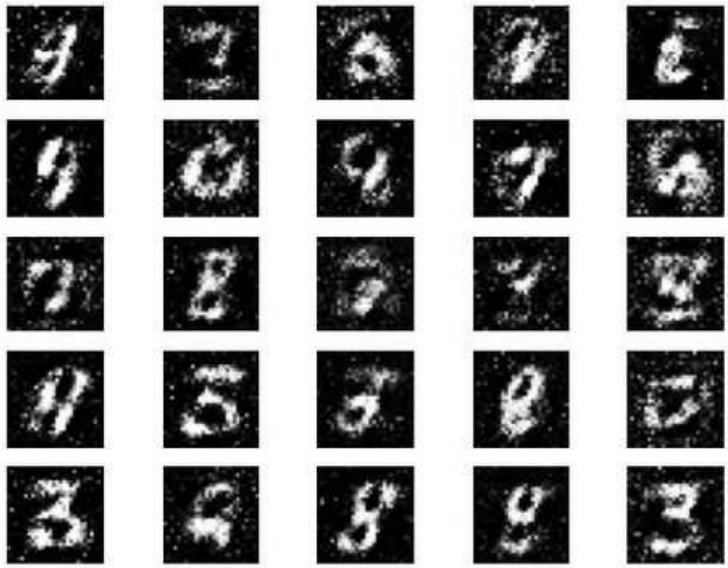

g

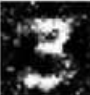

Generator step b
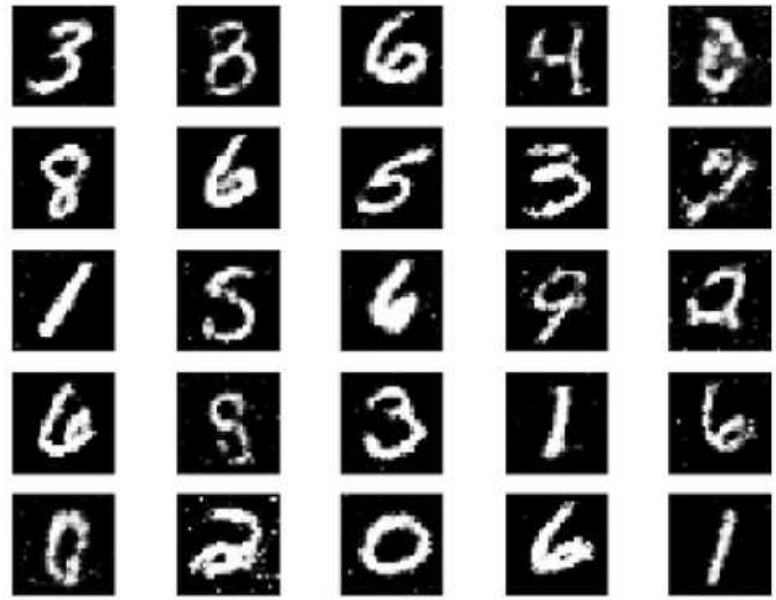

1

Figure 12

Top results of Generator test. 


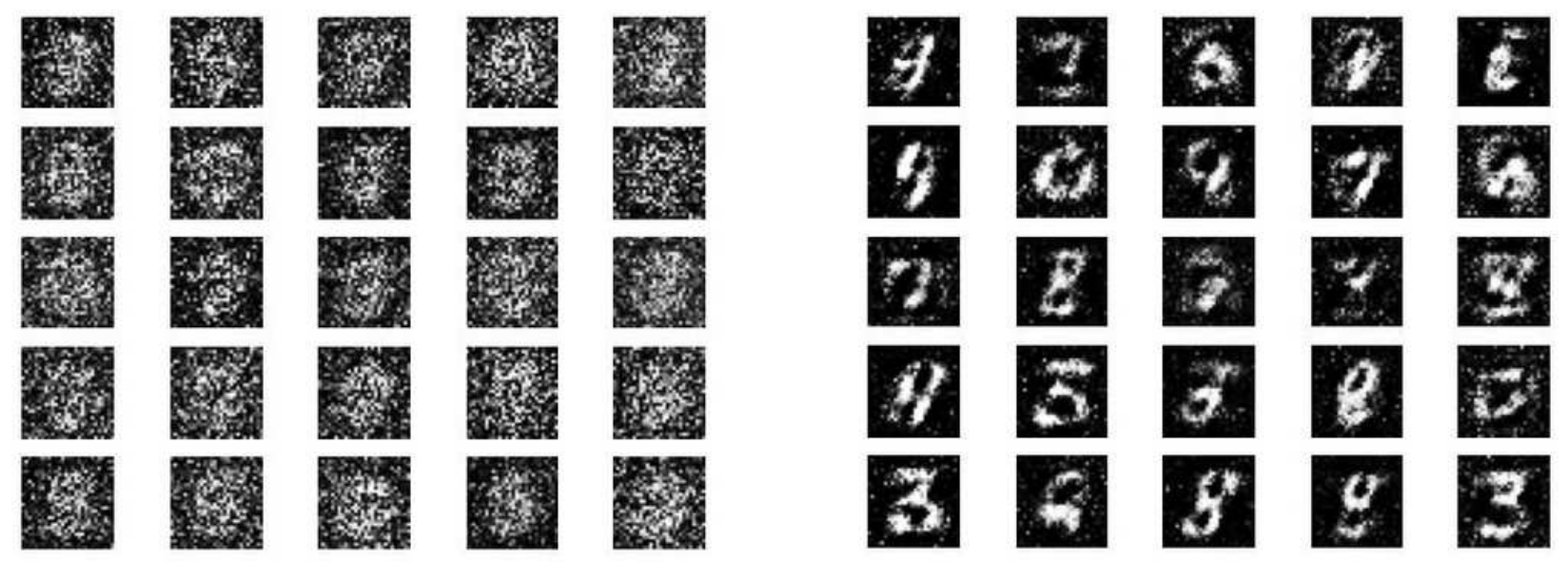

Generator step a

Generator step b

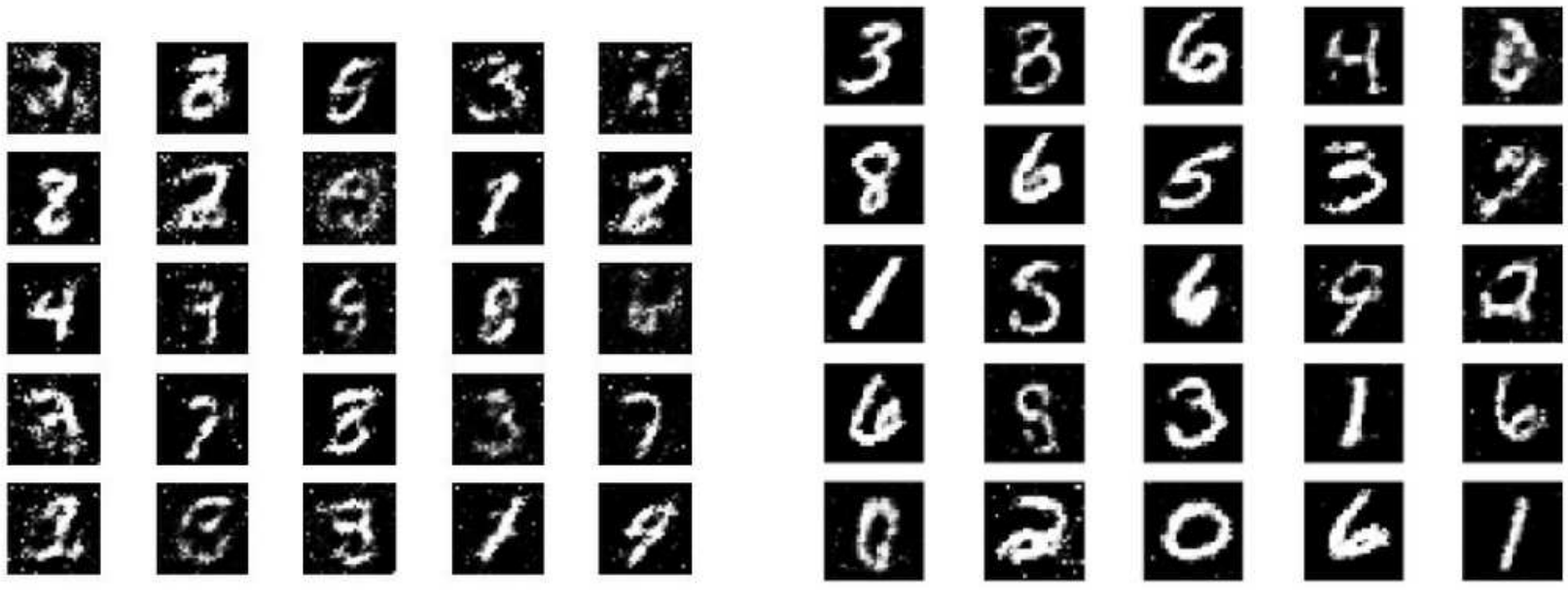

Generator step c

Generator final step

Figure 12

Top results of Generator test.

Image not available with this version 
Figure 13

Generator results of final testing.

\section{Image not available with this version}

Figure 13

Generator results of final testing. 